IASSNS-HEP-91/91

December 1991

\title{
Intersection Theory, Integrable Hierarchies And Topological Field Theory"̋
}

\author{
RoBbert DiJkgraAF" \\ School of Natural Sciences \\ Institute for Advanced Study \\ Princeton, NJ 08540
}

\begin{abstract}
In these lecture notes we review the various relations between intersection theory on the moduli space of Riemann surfaces, integrable hierarchies of KdV type, matrix models, and topological field theory. We focus in particular on the question why matrix integrals of the type considered by Kontsevich naturally appear as $\tau$-functions of integrable hierarchies related to topological minimal models.
\end{abstract}

*Lectures presented at the Cargèse Summer School on New Symmetry Principles in Quantum Field Theory, July 16-27, 1991.

${ }^{\dagger}$ Research supported by the W.M. Keck Foundation 


\section{IntRODUCTION}

The last two years have seen the emergence of a beautiful new subject in mathematical physics. It manages to combine a most exotic range of disciplines: two-dimensional quantum field theory, intersection theory on the moduli space of Riemann surfaces, integrable hierarchies, matrix integrals, random surfaces, and many more. The common denominator of all these fields is two-dimensional quantum gravity or, more general, low-dimensional string theory. Here the application of large- $N$ techniques in matrix models, that are used to simulate fluctuating triangulated surfaces [1]-[3], has led to complete solvability [4]-[8]. (See e.g. the review papers [9], and also the lectures of S. Shenker in this volume.) Shortly after the onset of the remarkable developments in matrix models, Edward Witten presented compelling evidence for a relationship between random surfaces and the algebraic topology of moduli space [10, 11]. This proposal involved a particular quantum field theory, known as topological gravity [12], whose properties were further established in [13, 14] and generalized to the so-called multi-matrix models in [15]-[19]. This subject can, among many other things, be considered as a fruitful application of quantum field theory techniques to a particular problem in pure mathematics, and as such is a prime example of a much bigger program, also largely due to Witten, that has been taking shape in recent years. It is impossible to do fully justice to this subject within the confines of these lecture notes. I will however make an effort to indicate some of the more startling interconnections.

I think it is fair to say that one of the most exciting recent developments in this field has been the work of the Russian mathematician Maxim Kontsevich [20]-22]. He did not only prove rigorously the first of a set of conjectures of Witten relating the calculus of intersection numbers on the moduli space of curves to integrable hierarchies of KdV type. In the process of that, he also derived a concrete 'matrix integral representation' of the string partition function. This is quite a curious result, since this matrix integral is in no obvious way related to the matrix models that initiated all these developments. In fact, one could say that Kontsevich's model is used to triangulate moduli space, whereas the original models triangulated Riemann surfaces.

Let me first state the main results that will be the revolving point of these notes. Our starting point will be the particular two-dimensional quantum field theory know as topological gravity. Let $\mathcal{O}_{n}$ denote the observables in this theory and $t_{n}$ the coupling constants to these operators. In the field theory we can consider correlation functions of the type

$$
\left\langle\mathcal{O}_{n_{1}} \cdots \mathcal{O}_{n_{s}}\right\rangle_{g}
$$

where $\langle\cdots\rangle_{g}$ denotes the expectation value on a (connected) surface with $g$ handles. These correlation functions represent, more or less by definition, characteristic numbers of the mod- 
uli space of Riemann surfaces. The string partition function $\tau(t)$ is defined as the generating functional of all possible correlation functions on all possible Riemann surfaces, not necessarily connected. That is, $\tau(t)$ has an asymptotic expansion of the form

$$
\tau(t)=\exp \sum_{g=0}^{\infty}\left\langle\exp \sum_{n} t_{n} \mathcal{O}_{n}\right\rangle_{g}
$$

Witten's conjecture states that, as anticipated in our notation,

$$
\tau(t) \text { is a tau-function of the KdV hierarchy. }
$$

We will explain the concept of a $\tau$-function in great detail in section 3 . It is a very useful and common notion in the study of integrable hierarchies. Essentially it implies that from $\tau(t)$ we can construct solutions to the famous Korteweg-de Vries equation

$$
\frac{\partial u}{\partial t}=u u^{\prime}+\frac{1}{6} u^{\prime \prime \prime}
$$

and all its generalizations. The relevant point is that $\tau(t)$ is completely calculable. This integrable structure was first found in the matrix model description of random surfaces or Euclidean quantum gravity, and so Witten's conjecture can be restated as the equivalence of quantum and topological gravity. The intuition behind this remarkable equivalence is some kind of poorly understood universality in theories of two-dimensional gravity.

The integral representation of $\tau(t)$ that emerges naturally in Kontsevich's solution involves the integral over a $N \times N$ Hermitian matrix $Y$ of the form

$$
\tau(Z)=\rho(Z)^{-1} \int d Y \cdot \exp \operatorname{Tr}\left[-\frac{1}{2} Z Y^{2}+\frac{i}{6} Y^{3}\right]
$$

Here $Z$ is a second $N \times N$ Hermitian matrix, and $\rho(Z)$ is the one-loop integral

$$
\rho(Z)=\int d Y \cdot \exp -\frac{1}{2} \operatorname{Tr} Z Y^{2}
$$

It is clear that $\tau(Z)$ is conjugation invariant, and so only depends on the eigenvalues $z_{1}, \ldots, z_{N}$ of $Z$. The relation between (1.2) and (1.5) requires a map from the matrix $Z$ to the coupling coefficients $t_{n}$. According to Kontsevich this map is given by

$$
t_{n}=-\frac{1}{n} \operatorname{Tr} Z^{-n}
$$

*In the case of two-dimensional gauge theories the equivalence of the topological and the non-topological model (also known as Yang-Mills theory) is much better understood due to recent work of Witten 23. 
As we will see, the matrix integral (1.5) has a natural expansion in Feynman diagrams and each diagram represents the integral over a particular cell in the moduli space of Riemann surfaces. It can therefore be considered as a finite-dimensional reduction of the path-integral of topological gravity.

Finally, there is a third and equivalent way to characterize $\tau(t)$, that naturally arises in topological field theory and in considerations of so-called loop operators. In this formulation a series of differential operators $L_{n}$ - quadratic in the parameters $t_{k}$ and their derivatives $\partial / \partial t_{k}$ - annihilate the string partition function [24, 25]

$$
L_{n} \cdot \tau=0, \quad n \geq-1
$$

These operators, that will be consider in greater detail in sections 4 and 7 , form part of a Virasoro algebra - they satisfy the commutation relations

$$
\left[L_{n}, L_{m}\right]=(n-m) L_{n+m}
$$

The constraints (1.8) can be used to derive recursion relations for the correlation functions and thus provide an alternative way to arrive at the asymptotic expansion for $\tau(t)$. From a computational perspective, (1.8) is perhaps the most practical characterization of $\tau(t)$.

There exist a whole hierarchy of generalizations of all these structures, where the results become more and more incomplete and conjectural as we move away from the original setting. The most simple generalization involves a choice of integer $p \geq 2$, with the case $p=2$ corresponding to intersection theory on moduli space. In the case there are well-defined candidates for the corresponding problem in algebraic geometry, the integrable hierarchy, and the matrix integral. As we will see this can naturally be considered the $A_{p-1}$ case in a series of models labeled by a simply-laced Lie group $G$, i.e., $G$ is of type $A_{n}, D_{n}, E_{6}, E_{7}$, or $E_{8}$. The most general case is based on an arbitrary two-dimensional topological field theory, and includes, among others, sigma-models with an almost complex target space. This field is known as topological string theory and its status as an integrable system is very unclear, to say the least.

Let me close this introduction with a short exposition of how this all is related to $c<1$ string theory, where one studies minimal conformal field theories on fluctuating surfaces. These minimal models are labeled by two (relative prime) integers $p$ and $q$ - the labeling $(p, q)$ is symmetric in $p$ and $q$. The models can be considered as isolated points in an ill-defined space of all two-dimensional quantum field theories coupled to gravity, as is symbolically illustrated in fig. 1. A neighbourhood of a minimal model consists of massive QFT's obtained by perturbation. The counting of physical observables is rather complicated [26], but there is a simple subsector - which does not involve the reparametrization ghosts - and in this 


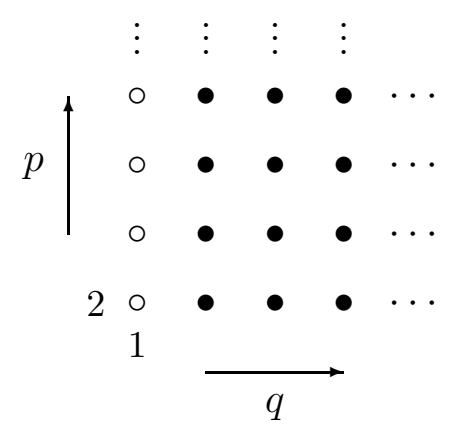

Fig. 1: The space of $c<1$ field theories coupled to quantum gravity contains as special point the $(p, q)$ minimal models. The models of type $(p, 1)$, here indicated by open dots, should be considered as topological field theories, and are the main subject of these notes.

subsector we find

$$
\text { \# states }=\frac{1}{2}(p-1)(q-1) .
$$

This finite number of states is the defining property of minimal models. Through the work of Kazakov [3], one can use matrix models to represent both the random surfaces and the conformal field theory by choosing the right critical behavior of the matrix potential. This leads to a uniform description of all models with identical $p$, where a chain of $(p-1)$ matrices suffices. The second number $q$ now labels the order of criticality. All models with fixed $p$ are related through the $p^{\text {th }} \mathrm{KdV}$ hierarchy [7], which moves horizontally in fig. 1. That is, their partition functions are given by the same $\tau$-function, evaluated at different values of the coupling coefficients $t_{n}$.

Models of type $(p, 1)$ are strictly speaking not well-defined conformal field theoriesaccording to the above formula they have zero physical fields. However, one can make sense out of these models as topological field theories, the so-called topological minimal models, and they will form the main topic of these lecture notes. Since the orbit of a topological model under the KdV flows will cover all other $(p, q)$ models with fixed $p$, this is in fact a very elegant and powerful way to study the general case. We will see that the behavior of many quantities simplifies dramatically at the topological point $q=1$, in particular the so-called Baker-Akhiezer function is exactly calculable, and this will lead to an explicit solution. As mentioned before this leads us again, through the work of Kontsevich, to matrix models, although of a very different kind. In fact, Kontsevich's generalized matrix model interpolates in a natural way between the models with different $p$-its allows 'vertical' deformations in fig. 1 - and can thus be seen as complementary to the original double-scaled matrix models.

In the following sections we will try to explain the various aspects of these beautiful but at first sight highly mysterious results and their many generalizations. We will focus in particular on the question why matrix integral s arise naturally in integrable hierarchies, 
a point that is also addressed in a number of recent publications [22, 27, 28]. These notes are organized as follows: We start our discussion with (generalized) intersection theory on moduli space in $\S 2$. The integrable hierarchies of KdV type that emerg e in that context are described in $\S 3$, which follows very closely the exposition of Segal and Wilson [29]. In $\S 4$ we show how the apparatus of Grassmannians can be applied to our specific problem and how all this is related to matrix integrals. The quantum field theories that have led us to these result $\mathrm{s}$ and conjectures first enter in $\S 5$. Here we only consider the general properties of topological field theories that obey factorization conditions. This is made much more explicit in $\S 6$, where various two-dimensional models are constructed, with particular emphasis on the so-called Landau-Ginzburg models. In $\S 7$ we finally discuss topological gravity, and more generally topological string theor y. Since we covered much of this material on a previous occasion [30], we will be here far less complete. In particular, the Virasoro constraints and thei $\mathrm{r}$ relation with loop equations will only be touched upon. Finally a point of caution: we have not always been extremely careful in our calculations with all 'irrelevant' numerical constants. We refer the reader to the literature for the factors of $i$ and $2 \pi$.

\section{INTERSECTION THEORY}

We start from the quantum field theory end, or more precisely from the finite dimensional cohomology problem to which the quantum field theory, by construction, can be reduced to. We will return to the description of the full quantum field theories in the sections 5-7.

\subsection{The Moduli SPACE OF CURVES}

Let us briefly recall some well-known facts about the moduli space of smooth complex curves or Riemann surfaces. A given topological surface $\Sigma$ with $g$ handles and $s$ marked points $x_{1}, \ldots, x_{s}$ can be made into a complex manifold by endowing it with a complex structure $J$, that is, a local linear map on the tangent bundle that satisfies $J^{2}=-1$ and the integrability condition $D J=0$. Two complex structures are considered equivalent if they are related by a diffeomorphism. This leaves us actually with a finite-dimensional space of inequivalent

complex structures on the surface, known as the moduli space $\mathcal{M}_{g, s}$. By Riemann-Roch this is a space of complex dimension

$$
\operatorname{dim} \mathcal{M}_{g, s}=3 g-3+s .
$$


(The moduli spaces $\mathcal{M}_{0, s}$ are only well-defined for $s \geq 3$, the exceptional case $\mathcal{M}_{1,0}$ equals $\mathcal{M}_{1,1}$ and is thus one-dimensional.) Moduli spaces are not everywhere smooth, since surfaces with accidental higher symmetry give rise to so-called orbifold points.

One way to think about the complex structure on $\Sigma$ is as the conformal class of a metric $h_{\mu \nu}$. Indeed, a metric defines a complex structure through

$$
J_{\mu}{ }^{\nu}=\sqrt{h} \epsilon_{\mu \lambda} h^{\lambda \nu}
$$

with $\epsilon_{\mu \nu}$ the Levi-Civita symbol, and it is a useful fact that all $J$ 's can be obtained in this way. Since the definition of $J$ is independent of local rescalings of $h_{\mu \nu}$, we can represent $\mathcal{M}_{g, s}$ alternatively as the space of metrics modulo local rescalings and diffeomorphisms.

The moduli space $\mathcal{M}_{g, s}$ has a boundary and there exists a direct, intuitive interpretation of the points at the boundary - they represent degenerate surfaces. There are basically two ways in which a surface can degenerate. If we think in terms of a conformal class of metrics, the surface can either form a node - or, equivalently, a long neck - or two marked points can collide. The boundary of $\mathcal{M}_{g, s}$ can be thought to lie at infinity. One would like to compactify the moduli space by adding points at infinity, not unlike how one compactifies the plane $\mathbf{R}^{n}$ to the $n$-dimensional sphere. In this case the points at infinity represent particular Riemann surfaces. The Deligne-Mumford or 'stable' compactification [31] adds three types of these points, as is illustrated in fig. 2.

(i) The process in which two points $x_{1}$ and $x_{2}$ 'collide' if $q=x_{1}-x_{2}$ tends to zero can (after a coordinate transformation $x \rightarrow x / q$ ) alternatively be described as the process in which a sphere, that contains $x_{1}$ and $x_{2}$ at fixed distance, pinches off the surface by forming a neck of length $\log q$. These two descriptions are fully equivalent, but the latter is actually more in the spirit of conformal field theory, since we see in an obvious way the operator product expansion emerge. It is also suggestive of another end point configuration. Here the natural final configuration is not simply the surface with $x_{1}=x_{2}$, but it consists of a separate sphere containing the points $x_{1}, x_{2}$ and a third point where the infinite long tube was attached, together with the original surface with one marked point less. In the stable compactification we add this configuration as limit point, which symbolically can be written as the process

$$
(g, s) \rightarrow(g, s-1)+(0,3) .
$$

Recall that the thrice-punctured sphere has a unique complex structure, so there are no moduli associated to it and this boundary component has codimension one. The crucial property of this compactification is that the points $x_{i}$ never come together. The two other degeneration modes are more straightforward.

(ii) If a cycle of non-trivial homology pinches, we replace the surface by a surface with one handle less and two extra marked points - the attachment points of the infinitely thin 
handle,

$$
(g, s) \rightarrow(g-1, s+2)
$$

(iii) In a similar spirit: in case a dividing cycle pinches, the resulting surface consists of two disconnected surfaces of genus $g^{\prime}$ and $g-g^{\prime}$, each having one extra puncture

$$
(g, s) \rightarrow\left(g, s^{\prime}+1\right)+\left(g-g^{\prime}, s-s^{\prime}+1\right) .
$$

It can be shown that this prescription makes $\mathcal{M}_{g, s}$ into a compact smooth (orbifold) space $\overline{\mathcal{M}}_{g, s}$. We now want to consider its cohomology ring $H^{*}\left(\overline{\mathcal{M}}_{g, s}\right)$. A particular set of elements has been considered by Mumford, Morita, and Miller [32]-34]. These classes are constructed as follows [10]. There exist $s$ natural line bundles $L_{1}, \ldots, L_{s}$ on the moduli space. The fiber of the bundle $L_{i}$ at a point $\Sigma \in \mathcal{M}_{g, s}$ is the cotangent space to the point $x_{i}$ on the surface $\Sigma$. These line bundles have first Chern classes that in de Rham cohomology are represented by the curvature $F_{i}$ of an arbitrary $U(1)$ connection on $L_{i}$. This defines for us the $2 n$-dimensional classes

$$
\sigma_{n}(i)=c_{1}\left(L_{i}\right)^{n} \in H^{2 n}\left(\mathcal{M}_{g, s}\right), \quad \sigma_{n}(i)=\underbrace{F_{i} \wedge \ldots \wedge F_{i}}_{n} .
$$

It is a non-trivial property that these are stable classes, i.e., that their definition actually extends to the compactified moduli space $\overline{\mathcal{M}}_{g, s}$. The reason is basically that the line bundles $L_{i}$ remain well-defined in the stable compactification, since the points $x_{i}$ will never collide. At infinity they remain at finite distance while being separated from the bulk of the surface on a 'frozen' 3-punctured sphere, which carries no moduli.

We can now define 'correlation functions' by pairing the wedge product of the $\sigma_{n}$ 's with the fundamental class of the moduli space

$$
\left\langle\sigma_{n_{1}} \cdots \sigma_{n_{s}}\right\rangle_{g} \equiv\left\langle\sigma_{n_{1}}(1) \cdots \sigma_{n_{s}}(s), \overline{\mathcal{M}}_{g, s}\right\rangle
$$

Of course, these intersection numbers are only non-zero if the degree of the total class equals the dimension of moduli space, which gives the 'charge conservation' condition

$$
\sum_{i=1}^{s}\left(n_{i}-1\right)=3 g-3
$$

Although the Chern classes $c_{1}\left(L_{i}\right)$ are in principle defined in integer cohomology, their intersection numbers will be in general rational numbers, due to the orbifold nature of the moduli space. 
The amazing result, first conjectured by Witten [10, 11] on the basis of the matrix model results and later proved rigorously by Kontsevich [20], states that all these intersection numbers are explicitly computable because their generating function obeys the equations of the $\mathrm{KdV}$ hierarchy. To state this result more precisely, it is convenient to rescale and relabel the classes $\sigma_{n}$ as

$$
\mathcal{O}_{2 n+1}=(2 n+1) ! ! \cdot \sigma_{n}, \quad n \geq 0
$$

After this substitution the theorem states that the string partition function

$$
\tau(t)=\exp \sum_{g=0}^{\infty}\left\langle\exp \sum_{k \text { odd }} t_{k} \mathcal{O}_{k}\right\rangle_{g}
$$

is a $\tau$-function of the $\mathrm{KdV}$ hierarchy. In this sense intersection theory on the (universal) moduli space is completely integrable. We note that due to the charge conservation condition (2.8), it is very easy to introduce a string coupling constant $\lambda$ that keeps track of the genus $g$ of the different surfaces that appear in the partition function $\tau(t)$. Indeed, if we rescale the coupling constants by

$$
t_{k} \rightarrow \lambda^{\frac{k}{3}-1} t_{k}
$$

we find

$$
\tau(t) \rightarrow \exp \sum_{g=0}^{\infty} \lambda^{2 g-2}\left\langle\exp \sum_{k \text { odd }} t_{k} \mathcal{O}_{k}\right\rangle_{g}
$$

Before we discuss the proof of the integrability of $\tau(t)$, let us first discuss some weaker results related to the 'trivial' nature of the classes $\sigma_{0}$ and $\sigma_{1}$.

\subsection{The PUnCtURE AND DILATON EQUATIONS}

The only consequence of the insertion of the 'puncture operator' $\sigma_{0}$ in a correlation function is that the same cohomology class is now integrated over $\overline{\mathcal{M}}_{g, s+1}$ instead of $\overline{\mathcal{M}}_{g, s}$. Since there is a natural projection map $\pi: \overline{\mathcal{M}}_{g, s+1} \rightarrow \overline{\mathcal{M}}_{g, s}$, where one simply forgets the position of the extra point, one might tend to conclude that the classes can be simply pull-backed and that thus the correlation function including $\sigma_{0}$ 's vanish by dimensional reasons. This is actually not true, due to subtleties at the divisor at infinity, where the forgotten point comes close to the other positions. This leads to extra corrections that can be expressed through the so-called puncture equation, due to Deligne [35], which is explained in more detail in [15],

$$
\left\langle\sigma_{0} \cdot \sigma_{n_{1}} \cdots \sigma_{n_{s}}\right\rangle_{g}=\sum_{i=1, n_{i} \neq 0}^{s}\left\langle\sigma_{n_{1}} \cdots \sigma_{n_{i}-1} \cdots \sigma_{n_{s}}\right\rangle_{g}
$$


This relation can be used to eliminate all $\sigma_{0}$ 's from a particular correlation function. It is of course only valid if both sides of the equation are well-defined, which is not the case in genus zero with less than three insertions. In fact, there we have the additional relation

$$
\left\langle\sigma_{0} \sigma_{0} \sigma_{0}\right\rangle_{0}=1
$$

a simple consequence of $\mathcal{M}_{0,3}=$ point.

A similar result can be obtained for the insertions of the next operator in line- the 'dilaton operator' $\sigma_{1}$. Since $\sigma_{1}$ is the first Chern class $c_{1}(L)$ it can be shown to calculate the degree of the canonical line bundle of a genus $g$ surface with $s$ punctures [11. The dilaton equation exactly expresses this result

$$
\left\langle\sigma_{1} \cdot \sigma_{n_{1}} \cdots \sigma_{n_{s}}\right\rangle_{g}=(2 g-2+s)\left\langle\sigma_{n_{1}} \cdots \sigma_{n_{s}}\right\rangle_{g}
$$

Here again we must be careful if the right-hand side is ill-defined. This happens in genus one with no punctures. Here one finds

$$
\left\langle\sigma_{1}\right\rangle_{1}=\frac{1}{24}
$$

The above equations allow one to eliminate all operators $\sigma_{0}$ and $\sigma_{1}$. Actually this, together with the selection rule (2.8), suffices to reduce any intersection number in genus zero or one to (2.14) and (2.16) respectively. On the sphere the final result reads

$$
\left\langle\sigma_{n_{1}} \cdots \sigma_{n_{s}}\right\rangle_{0}=\frac{\left(n_{1}+\ldots+n_{s}\right) !}{n_{1} ! \ldots n_{s} !}
$$

On the torus it is more difficult to give a closed form expression, but one way to state the result is by means of generating functions

$$
\left\langle\exp \sum_{n} t_{n} \sigma_{n}\right\rangle_{1}=\frac{1}{24} \log \left\langle\sigma_{0}^{3} \cdot \exp \sum_{n} t_{n} \sigma_{n}\right\rangle_{0}
$$

In this fashion one can verify the KdV relations by hand at low genus. Similar verification have been done by Witten [1] in genus two and Horne [36] in genus three, using results of Mumford [32] and Faber [37] respectively. 


\subsection{Kontsevich's CALCUlation}

There exists a very explicit realization of the moduli spaces $\mathcal{M}_{g, s}$ for $s>0$ due to the work of Strebel [38], Penner [39], and others, that naturally arises in open string field theory. Recall that we can think of complex structures in terms of conformal classes of metrics. In open string field theory Riemann surfaces are built out of flat strips (propagators) with fixed width and flexible lengths $\ell_{a}$ by glueing them together with a three-point vertex, in such a way that all the curvature is localized in the mid-point of the vertex. With $3 k$ propagators and $2 k$ vertices one produce a surface of Euler number $\chi=2-2 g-s=-k$. The corresponding Feynman diagram is called a ribbon or fat graph. Some examples are sketched in fig. 3. These surface have by construction at least one boundary. We can associate a closed surface with punctures to a ribbon graph $\Gamma$ by glueing onto the boundary components $C_{1}, \ldots, C_{s}$ infinitely long cylinders - which are conformally equivalent to punctured disks. We have $6 g-6+3 s$ real variables $\ell_{a}$ parametrizing the surface, $s$ of which correspond to the total lengths of the boundaries while the other variables parametrize a top-dimensional simplex in the moduli space $\mathcal{M}_{g, s}$. It is a powerful result that we can actually obtain any point of the moduli space once and only once by considering all connected graphs $\Gamma$ of the appropriate Euler number.

We can define a 'path-integral' whose expansion in Feynman diagrams naturally produces the graphs $\Gamma$. The fundamental idea, basically due to 't Hooft [40], is to use an Hermitian $N \times N$ matrix $Y$ to represent the double line propagator, appropriate for a ribbon graph. Indeed, the matrix integral

$$
Z=\int d Y \cdot \exp \frac{1}{\lambda} \operatorname{Tr}\left[-\frac{1}{2} Y^{2}+\frac{1}{3} Y^{3}\right]
$$

has an expansion

$$
\log Z=\sum_{\Gamma} \frac{1}{n(\Gamma)} \lambda^{2 g-2+s} N^{s}
$$

where we sum over all connected graphs $\Gamma ; n(\Gamma)$ is the order of the automorphism group of the graph $\Gamma, \lambda$ is the string coupling constant. $Z$ simply counts all top-dimensional cells exactly once, and thus represents an 'integral' over moduli space.

Kontsevich has used this 'open string field theory' description of moduli space to calculate the intersection numbers of the classes $\sigma_{n}$. The first step is to write the Chern classes $c_{1}\left(L_{i}\right)$ in terms of the lengths $\ell_{a}$ 's. Let us concentrate on one boundary component $C_{i}$ of the surface. It has total length

$$
p_{i}=\sum_{a \in I_{i}} \ell_{a},
$$

where $I_{i}$ labels all propagators that contribute to the boundary $C_{i}$. According to Kontsevich the subdivision of $p_{i}$ in the $\ell_{a}$ can be used to define a natural connection on the circle bundle, 
or actually the polygon bundle, $L_{i}$ associate to the boundary. The first Chern class of this line bundle $L_{i}$ is the given by the curvature of this connection, and takes the form

$$
c_{1}\left(L_{i}\right)=\sum_{a, b \in I_{i}} d \theta_{a} \wedge d \theta_{b}, \quad \theta_{a}=\ell_{a} / p_{i}
$$

We now want to perform the integral of these Chern classes over moduli space. We do not have the space here to reproduce the complete calculation, which has many deep subtleties. Let us briefly indicate the crucial steps. First, one considers not the classes $\sigma_{n}$ but the 'loop operators'

$$
w(z)=\int d p e^{-z p} \exp \frac{1}{2} p^{2} c_{1}(L)=\sum_{k \text { odd }} \frac{1}{k} z^{-k} \mathcal{O}_{k} .
$$

One then evaluates the correlation function of these operators $w(z)$ for fixed genus $g$. This is an integral over both $\mathcal{M}_{g, s}$ and the boundary lengths $p_{1}, \ldots, p_{s}$. According to Kontsevich this integral becomes elementary when expressed in terms of the coordinates $\ell_{a}$. With the two-form $\Omega$ defined by

$$
\Omega=\sum_{i=1}^{s} \frac{1}{2} p_{i}^{2} c_{1}\left(L_{i}\right),
$$

we see that the integral contains indeed the simple measure factor

$$
e^{\Omega} \cdot d p_{1} \wedge \ldots \wedge d p_{s}=c \cdot \prod_{a} d \ell_{a}
$$

Surprisingly the constant $c$ does not depend on the graph $\Gamma$. One simply finds $c=2^{k}$ for a surface with Euler characteristic $\chi=-k$ (see Appendix $\mathrm{C}$ of [22]), and the calculation reduces to

$$
\left\langle w\left(z_{1}\right) \cdots w\left(z_{s}\right)\right\rangle_{g}=\sum_{\Gamma} \frac{2^{k}}{n(\Gamma)} \int[d \ell] \prod_{i, a \in I_{i}} e^{-z_{i} \ell_{a}}
$$

which can be directly evaluated to give

$$
\left\langle w\left(z_{1}\right) \cdots w\left(z_{s}\right)\right\rangle_{g}=\sum_{\Gamma} \frac{2^{-2 k}}{n(\Gamma)} \prod_{\text {prop }} \frac{2}{z_{i}+z_{j}} .
$$

Here the summation is over all diagrams $\Gamma$ that contribute to $\mathcal{M}_{g, s}$ and the product is over all propagators. A factor $2 /\left(z_{i}+z_{j}\right)$ is included when the two sides of the propagator form part of the loops $C_{i}$ and $C_{j}$. This is but a small modification of the Feynman rules of the integral (2.19). We only have to change the quadratic part. The factor $2^{-2 k}$ can be absorbed in the weight of the cubic vertex, or in the string coupling constant, if one wishes. In fact, 
we arrive in this way directly at a representation of the generating functional of the loop operator correlation functions

$$
\tau\left(z_{1}, \ldots, z_{N}\right)=\exp \sum_{g=0}^{\infty}\left\langle\exp \sum_{i=1}^{N} w\left(z_{i}\right)\right\rangle_{g}
$$

by means of the matrix integral

$$
\tau(Z)=\rho(Z)^{-1} \int d Y \cdot \exp \operatorname{Tr}\left[-\frac{1}{2} Z Y^{2}+\frac{1}{6} Y^{3}\right]
$$

Here $z_{1}, \ldots, z_{N}$ are the eigenvalues of the $N \times N$ Hermitian matrix $Z$. We have divided by the one-loop integral

$$
\rho(Z)=\int d Y \cdot \exp -\frac{1}{2} \operatorname{Tr} Z Y^{2}=\prod_{i, j}\left(z_{i}+z_{j}\right)^{-\frac{1}{2}}
$$

in order to obtain the correct asymptotic expansion. We see from (2.23) that (2.29) corresponds to the following parametrization of the coupling constants $t_{k}$

$$
t_{k}=\sum_{i=1}^{N} \frac{1}{k} z_{i}^{-k}=\frac{1}{k} \operatorname{Tr} Z^{-k}
$$

We will learn in $\S 3$ that this is a very natural parametrization from the point of view of the $\mathrm{KdV}$ hierarchy. The matrix integral can be made absolutely convergent by putting a factor of $i$ in front of the cubic term in the action. One easily sees that, by charge conservation, this is equivalent to a redefinition $t_{k} \rightarrow-t_{k}$. In this way we recover Kontsevich's result [20] that we mentioned in the Introduction.

\subsection{Witten's Generalizations}

Witten has formulated in [18, 19] a generalization of the above intersection theory, which involves the choice of an integer $p \geq 2$, and extra quantum numbers $k_{i}=0, \ldots, p-2$ for each of the points $x_{i}$. Our observables are now of the form $\sigma_{n, k}$, and their correlation functions are defined as

$$
\left\langle\sigma_{n_{1}, k_{1}} \cdots \sigma_{n_{s}, k_{s}}\right\rangle_{g} \equiv p^{-g}\left\langle\sigma_{n_{1}}(1) \cdots \sigma_{n_{s}}(s) \cdot c_{D}(V), \overline{\mathcal{M}}_{g, s}^{\prime}\right\rangle
$$

Let us explain the different objects that enter in this definition. We have already met the Mumford classes $\sigma_{n}$. The cohomology class $c_{D}(V)$ is the top dimensional Chern class of a 
complex $D$-dimensional vector bundle $V$ over moduli space. The definition of $V$ depends on the numbers $p$ and $k_{i}$ as follows [18, 19]. Let $S$ be the line bundle $K^{p-1} \otimes_{i} \mathcal{O}\left(z_{i}\right)^{k_{i}}$. That is, its section are $p-1$ forms that can have poles of order $k_{i}$ at the point $x_{i}$. The degree of $S$ is given by

$$
\operatorname{deg}(S)=(p-1)(2 g-2)+\sum_{i=1}^{s} k_{i} .
$$

If this degree is a multiple of $p$ we can define another line bundle $T$ as the $p^{\text {th }}$ root of $S$

$$
T^{\otimes p}=S, \quad \text { if } \quad \operatorname{deg}(S) \equiv 0(\bmod p) .
$$

Actually there are $p^{2 g}$ choices, and this defines a branched covering of $\mathcal{M}_{g, s}$ that is denoted as $\mathcal{M}_{g, s}^{\prime}$. The fiber $V_{\Sigma}$ of the vector bundle $V$ at a point $\Sigma \in \mathcal{M}_{g, s}$ is defined as the space of holomorphic section of $T$

$$
V_{\Sigma}=H^{0}(\Sigma, T), \quad \text { if } \quad H^{1}(\Sigma, T)=0
$$

This is true if the degree of $T$ is large enough. (See 19 for a more precise definition that takes into account that $H^{1}(\Sigma, T)$ may not always vanish.) In that case Riemann-Roch tells us that the fiber of $V$ has complex dimension

$$
D=d(g-1)+\sum_{i=1}^{s} q_{i}
$$

where we introduced the new notations

$$
d=\frac{p-2}{p}, \quad q_{i}=\frac{k_{i}}{p}
$$

By construction $D$ is always a non-negative integer. Combining all these ingredients we see that the correlation functions are only non-vanishing if the following charge conservation holds

$$
\sum_{i=1}^{s}\left(n_{i}+q_{i}-1\right)=(3-d)(g-1)
$$

It is sometimes convenient to denote the operators $\sigma_{n, k}$ slightly different. From the definition it is clear that $\sigma_{n, k}$ is more or less the tensor product of a Chern class and 'fractional class' related to the bundle $T$. That is, we would like to write symbolically

$$
\sigma_{n, k} \sim \sigma_{n} \otimes \phi_{k}
$$


with $\phi_{k}$ an operator of fractional charge $q=k / p$. We will make more sense out of this notation later on, but at this moment we only want to emphasize the special nature of the fields $\sigma_{0, k}$ that we also write as $\phi_{k}$. These operators do not depend on the Mumford classes, only on the top Chern class $c_{D}(V)$, and we will refer to these operators as 'primary.' The primary correlation functions

$$
\left\langle\phi_{k_{1}} \cdots \phi_{k_{s}}\right\rangle_{g} \equiv p^{-g}\left\langle c_{D}(V), \overline{\mathcal{M}}_{g, s}^{\prime}\right\rangle
$$

can be studied in their one right, and this is actually what we will undertake in $\S 6$. The primary fields $\phi_{k}$ can be considered as the observables in a separate topological field theory, and the combinations with the Mumford classes can be described as the coupling to topological gravity. This line of thought suggests many other generalizations, as we will see in the second half of these notes.

To state the generalized conjectures about the general correlation functions (2.32) we have again to renormalize our operators, this time as

$$
\mathcal{O}_{n p+k+1}=(n p+k+1)((n-1) p+k+1) \cdots(k+1) \cdot \sigma_{n, k} .
$$

So, the operators $\mathcal{O}_{n}$ in this case are labeled by a positive integer $n$ that satisfies $n \neq 0(\bmod$ $p)$. The claim is that $\tau(t)$ as defined by

$$
\tau(t)=\exp \sum_{g=0}^{\infty}\left\langle\exp \sum_{n} t_{n} \mathcal{O}_{n}\right\rangle_{g}
$$

is now a $\tau$-function of the $p^{\text {th }}$ generalized $\mathrm{KdV}$ hierarchy. Actually there is also a simple candidate matrix integral representation of $\tau(t)$ that we will meet in the next section-we basically replace the cubic interaction by a vertex of order $p+1$. In $\S \S 6$ and 7 we will consider the quantum field theory that lies behind this conjecture.

It is not difficult to find the generalizations of the puncture and dilaton equations in this setup. These are again related to the special role of the operators $\sigma_{0,0}$ and $\sigma_{1,0}$. For instance, one easily obtains the generalized puncture equation

$$
\left\langle\sigma_{0,0} \cdot \sigma_{n_{1}, k_{1}} \cdots \sigma_{n_{s}, k_{s}}\right\rangle_{g}=\sum_{i=1, n_{i} \neq 0}^{s}\left\langle\sigma_{n_{1}, k_{1}} \cdots \sigma_{n_{i}-1, k_{i}} \cdots \sigma_{n_{s}, k_{s}}\right\rangle_{g}
$$

The genus zero correction reads in this case

$$
\left\langle\sigma_{0,0} \sigma_{0, i} \sigma_{0, p-i-2}\right\rangle_{0}=1
$$


or, in the notation (2.41),

$$
\left\langle\mathcal{O}_{1} \mathcal{O}_{i} \mathcal{O}_{j}\right\rangle_{0}=i j \cdot \delta_{i+j, p}
$$

A dilaton equation, completely analogous to (2.15), can also be derived, with as only new ingredient the expectation value [19]

$$
\left\langle\mathcal{O}_{p+1}\right\rangle_{1}=(p+1)\left\langle\sigma_{1,0}\right\rangle_{1}=\frac{p^{2}-1}{24} .
$$

As a little warm-up for our discussion in $\S 4.4$, we mention here that the puncture equation (2.43) can be written as a linear partial differential equation for the string partition function $\tau(t)$

$$
L_{-1} \cdot \tau=0,
$$

where $L_{-1}$ is the differential operator

$$
L_{-1}=-\frac{\partial}{\partial t_{1}}+\sum_{k=p+1}^{\infty} k t_{k} \frac{\partial}{\partial t_{k-p}}+\frac{1}{2} \sum_{i+j=p} i j t_{i} t_{j} .
$$

This identity follows immediately, once it is realized that in a generating functional a derivative $\partial \tau / \partial t_{k}$ represent an insertion of the operator $\mathcal{O}_{n}$ in each correlation function, whereas a multiplication $t_{n} \cdot \tau$ eliminates the same operator. The last term on the RHS represents the correction for the exceptional case (2.45). A little further experimentation, which we leave as an exercise to the reader, shows that a particular linear combination of the dilaton equation and the charge conservation condition (2.38) leads to a similar differential equation

$$
L_{0} \cdot \tau=0,
$$

with $L_{0}$ given by

$$
L_{0}=-\frac{\partial}{\partial t_{p+1}}+\sum_{k=1}^{\infty} k t_{k} \frac{\partial}{\partial t_{k}}+\frac{p^{2}-1}{24} .
$$

These two equations are but the beginning of a full tower of equations, as we alluded to in the Introduction, and we will discus this at greater lengths in $§ 4.4$.

Let us end this brief description with some specializations. The case $p=2$ should reduce to 'standard' intersection theory on moduli space, which is indeed the case, since the bundle $V$ is now zero-dimensional by (2.36). Another interesting case, although somewhat outside our range of definition, is the case $p=-1$ [18], where (with all $\left.m_{i}=0\right) V$ can be identified with the bundle of quadratic differentials, i.e., the cotangent bundle $T^{*} \mathcal{M}_{g, 0}$ to moduli space. 
In this case the top Chern class $c_{D}(V)$ actually represents, up to a sign, the Euler class of moduli space and we can calculate

$$
\langle 1\rangle_{g}=\left\langle c_{D}(V), \overline{\mathcal{M}}_{g, 0}\right\rangle=(-1)^{3 g-3} \chi_{g, o},
$$

where we used the notation $\chi_{g, s}$ for the (virtual) Euler characteristic of the moduli space $\mathcal{M}_{g, s}$. Actually due to the work of Harer and Zagier [41] and, in particular, Penner [39] we know a concrete realization of the generating functional of these Euler numbers. If $Y$ is again an $N \times N$ matrix then the following matrix integral generates all Euler characteristics

$$
\log \int d Y \cdot \exp \frac{1}{\lambda} \operatorname{Tr}[\log (1-Y)-Y]=\sum_{g, s} N^{s} \lambda^{2 g-2+s} \chi_{g, s}
$$

The proof of this result is based on the observation that this matrix integral simply counts all $k$-simplices in the cell decomposition of $\mathcal{M}_{g, s}$ weighted with a factor $(-1)^{k}$.

\section{Integrable Hierarchies}

In this section we will change our point of view and investigate in greater detail the integrable hierarchies that have emerged in solvable string theories and intersection theory. The following is basically a self-contained elementary exposition of a number of familiar techniques in the theory of integrable systems, and some readers may wish to skip this part. A good survey of these matters is given in [29], which we will follow closely. We gladly acknowledge that a much higher level of sophistication can be found in the extensive literature on integrable systems. All these techniques will find their due applications in $\S 4$.

\subsection{The generalized KdV and KP hierarchies}

We will first describe the so-called generalized KdV hierarchy in its scalar Lax formulation. Let $x$ be a real variable and let $D$ denote the operator

$$
D=\frac{\partial}{\partial x}
$$

For a given integer $p \geq 2$ we will consider the general differential operator of order $p$

$$
L=D^{p}+\sum_{i=0}^{p-2} u_{i}(x) D^{i} .
$$


Here the coefficients $u_{i}(x)$ are a priori arbitrary functions in the variable $x$. Note that by conjugation the operator $L$ can always be put in the above form with vanishing term of order $p-1$. We now consider the flow of $L$ in an infinite set of 'times' $t_{1}, t_{2}, \ldots$ as generated by Hamiltonians $H_{1}, H_{2}, \ldots$

$$
\frac{\partial L}{\partial t_{n}}=\left[H_{n}, L\right]
$$

In order to be able to consider the operator $L(t)$ as a simultaneous functions of all parameters $t_{n}$ the Hamiltonians - which in general are time-dependent themselves - are required to generate commuting flows, and therefore satisfy the zero-curvature relation, or Zakharov-Shabat equations,

$$
\frac{\partial H_{m}}{\partial t_{n}}-\frac{\partial H_{n}}{\partial t_{m}}+\left[H_{m}, H_{n}\right]=0 .
$$

There is a unique basis for such commuting Hamiltonians; they are given by the non-negative part of the fractional powers of $L$

$$
H_{n}=\left(L^{n / p}\right)_{+}
$$

Let us explain this notation. We can define fractional powers of $L$ as Laurent series in the differential $D$. So in general these are so-called pseudo-differential operators, i.e., operators of the form

$$
A=\sum_{i=-\infty}^{n} a_{i}(x) D^{i} .
$$

The restriction of this sum to only the non-negative powers of $D$ is denoted by $A_{+}$

$$
A_{+}=\sum_{i=0}^{n} a_{i}(x) D^{i}
$$

One similarly defines $A_{-}=A-A_{+}$. We take this occasion to introduce another useful concept: the residue of a pseudo-differential operator. This is defined as the coefficient of $D^{-1}$ in the Laurent expansion (3.6) of $A$

$$
\operatorname{res} A=a_{-1}(x)
$$

We will apply this notion in a moment.

It is not difficult to explicitly verify that the flows (3.5) indeed commute. These hierarchies, describing the evolution of an $p^{\text {th }}$ order differential operator, are known as the generalized KdV or Gelfand-Dikii 42 hierarchies. Since the operator $L$ satisfies (3.3), its coefficients can be considered to be functions of both the coordinate $x$ and the times $t_{n}$. In fact, this is a somewhat redundant parametrization, because $x$ can be identified with $t_{1}$, since we have

$$
H_{1}=\left(L^{1 / p}\right)_{+}=D
$$


Furthermore, if $n$ is a multiple of $p$, the flows $t_{n}$ are trivial

$$
\frac{\partial L}{\partial t_{n}}=0, \quad \text { if } n=q \cdot p
$$

since in that case $H_{n}=L^{q}$ which clearly commutes with $L$.

As an example we can consider the simplest case $p=2$, where we have

$$
L=D^{2}+u(x)
$$

the Hamiltonian of a particle in a potential $u(x)$. One arrives at the original Kortewegde Vries equation by considering the first non-trivial flow of this operator, as generated by $H_{3}=D^{3}+\frac{3}{2} u D+\frac{3}{4} u^{\prime}$,

$$
\frac{\partial L}{\partial t_{3}}=\frac{\partial u}{\partial t_{3}}=\left[H_{3}, L\right]=u \frac{\partial u}{\partial x}+\frac{1}{6} \frac{\partial^{3} u}{\partial x^{3}}
$$

All the KdV flows can be considered special cases of the more general KP hierarchy which is defined as follows. Let $Q$ denote the unique $p^{\text {th }}$ root of the differential operator $L$

$$
Q=L^{1 / p}
$$

that has an expansion of the form

$$
Q=D+\sum_{i=1}^{\infty} q_{i} D^{-i}
$$

Then $Q$ satisfies a similar set of evolution equations as $L$

$$
\frac{\partial Q}{\partial t_{n}}=\left[H_{n}, Q\right], \quad H_{n}=Q_{+}^{n}
$$

These can be considered as the defining equations of the KP hierarchy, if $Q$ is now taken to be an arbitrary pseudo-differential operator with an expansion (3.14). One specializes to the $p^{t h} \mathrm{KdV}$ case by imposing that $Q^{p}=L$ is a pure differential operator, that is, by requiring

$$
Q_{-}^{p}=0
$$

The KP hierarchy can be slightly rewritten by introducing yet another pseudo-differential operator $K$ through

$$
Q=K D K^{-1}
$$


where $K$ has an expansion of the form

$$
K=1+\sum_{i=1}^{\infty} a_{i} D^{-i}
$$

It satisfies the equation

$$
\frac{\partial K}{\partial t_{n}}=-Q_{-}^{n} \cdot K
$$

We end this long list of definitions with a side remark. It is rather straightforward to take the 'classical' limit, also known as the dispersionless KdV hierarchy 43, 44] (see also the lectures of I. Krichever in these proceedings.) We simply put $\hbar$ in all the obvious places and take the $\hbar \rightarrow 0$ limit. In this fashion the differential $D$ becomes the classical momentum $y$ conjugate to $x$, and the differential operator $L$ becomes a function, polynomial in $y$, on the phase space $(y, x)$ with symplectic form $d y \wedge d x$,

$$
L(y, x)=y^{p}+u_{p-2}(x) y^{p-2}+\ldots+u_{0}(x) .
$$

All commutators reduce to Poisson brackets, in particular we have

$$
\frac{\partial L}{\partial t_{n}}=\left\{H_{n}, L\right\}=\frac{\partial H_{n}}{\partial y} \frac{\partial L}{\partial x}-\frac{\partial H_{n}}{\partial x} \frac{\partial L}{\partial y}
$$

We will see that, in terms of the string partition function, $\hbar$ corresponds to the string coupling constant $\lambda$, and that the classical limit implies the restriction to genus zero. We will return to these matters in $\S 6.4$.

\subsection{The $\tau$-FUnCtion AND THE BAKER-AKHIEZER FUnCTION}

To a solution $L(t)$ of the KdV equations (3.3) one can associate a so-called tau-function $\tau(t)$. It is in several ways related to the differential operator $L$. A particularly concrete connection, though perhaps not the most insightful, is given in terms of the operator $K$ defined in (3.17)

$$
\operatorname{res} K=-\frac{\partial}{\partial x} \log \tau
$$

or, equivalently, in terms of the Lax operator $L$

$$
\operatorname{res} L^{n / p}=\frac{\partial^{2}}{\partial x \partial t_{n}} \log \tau \text {. }
$$


Note that if $i=1, \ldots, p-1$,

$$
\operatorname{res} L^{i / p}=\frac{i}{p} \cdot u_{p-i-1}+\ldots
$$

where the ellipses indicate polynomials in the coefficients $u_{j}$ and there derivatives $u_{j}^{\prime}, u_{j}^{\prime \prime}, \ldots$ with respect to $x=t_{1}$, all satisfying $j>p-i-1$. Thus the transformations from the coefficients $u_{i-1}$ to the residues res $L^{i / p}$ is seen to be upper-triangular and thus invertible. In this sense the operator $L$ can be reconstructed from the $\tau$-function.

The opposite problem, how to construct $\tau$ out of $L$, will be considered in greater detail in §3.4. A crucial ingredient in this construction is played by the eigenfunctions $\psi$ of $L$. Since the operator $L$ depends itself on the times $t_{n}$, these eigenfunctions depend on the coordinate $x=t_{1}$, the other variables $t_{n}$ and the eigenvalue that we choose to parametrize as $z^{p}$. With the understanding that $x$ can be identified with $t_{1}$ we will write the eigenfunction as $\psi(t, z)$. Its defining equation is

$$
L \psi(t, z)=z^{p} \psi(t, z) .
$$

The function $\psi(t, z)$ is called the Baker-Akhiezer function. It satisfies the Schrödinger equations

$$
\frac{\partial \psi}{\partial t_{n}}=H_{n} \psi
$$

We will assume it is normalized such that

$$
\psi(t, z)=g(t, z) \cdot \eta(t, z), \quad g(t, z)=\exp \sum_{n} t_{n} z^{n}
$$

where $\eta(t, z)$ has an asymptotic expansion in $z^{-1}$ of the form

$$
\eta(t, z)=1+\sum_{i=1}^{\infty} a_{i}(t) z^{-i}
$$

Note that $\eta=1$ corresponds to the trivial case $L=D^{p}$. In fact, since in the general case $L=K D^{p} K^{-1}$, one can write $\psi$ as

$$
\psi(t, z)=K \cdot g(t, z)
$$

which shows that the coefficients $a_{i}$ in (3.17) and (3.28) are indeed identical. 


\subsection{GRassmannians And FERmions}

There is a beautiful way to generate solutions to the KP hierarchy using Grassmannians or equivalently two-dimensional free (chiral) fermions. This method, originally due to Sato [45, 46], is further explored and exposed in the beautiful paper by Segal and Wilson [29].

The basic idea is the following. For every operator $L(t)$ we can consider its Baker-Akhiezer function $\psi(t, z)$ as defined in (3.25). For fixed value of the $t_{n}$ 's, and after an appropriate analytic continuation in $z$, the function $\psi(z)$ can be seen as a wave function in the Hilbert space $H=L^{2}\left(S^{1}\right)$ with $z$ the coordinate on the unit circle. When the function $\psi(t, z)$ evolves in time it will sweep out a linear subspace $W$ of $H$

$$
\psi(t, z) \in W \subset H
$$

The method of Grassmannians constructs the solution $\psi(t, z)$ out of a given linear space $W \subset H$.

The wave functions in $H$ can be given the interpretation of one particle fermion states, with Hamiltonian $z \partial_{z}$. We have a basis of eigenstates $\left\{z^{n}\right\}$ in $H$ with energy $n \in \mathbf{Z}$, and a natural polarization

$$
H=H_{+} \oplus H_{-}
$$

in terms of positive and negative energy states. That is, $H_{+}$has a basis $z^{0}, z^{1}, \ldots$, whereas $H_{-}$is spanned by $z^{-1}, z^{-2}, \ldots$. The Grassmannian $\operatorname{Gr}(H)$ is defined as the space of subsets $W$ that (in some precise sense) are comparable to $H_{+}$. The Grassmannian can be seen as the second quantized fermion Fock space. Indeed, to the subspace $H_{+}$we can associate the semi-infinite wedge product

$$
|0\rangle=z^{0} \wedge z^{1} \wedge z^{2} \wedge \ldots
$$

which is just the Dirac vacuum, where - with an unfortunate choice of conventions - we filled all 'positive energy states.' If $w_{0}, w_{1}, \ldots$ is a basis for the subspace $W$, we can similarly construct the state

$$
|W\rangle=w_{0} \wedge w_{1} \wedge w_{2} \wedge \ldots
$$

Recall that in this notation the fermion fields have an expansion

$$
\psi(z)=\sum_{n} \psi_{n} z^{n}, \quad \psi^{*}(z)=\sum_{n} \psi_{n}^{*} z^{n}
$$

and act on the states as

$$
\psi_{n}=\frac{\partial}{\partial z^{n}}, \quad \psi_{n}^{*}=z^{-n} \wedge
$$


These operators obviously satisfy the canonical anti-commutation relations

$$
\left\{\psi_{n}, \psi_{m}\right\}=\left\{\psi_{n}^{*}, \psi_{m}^{*}\right\}=0, \quad\left\{\psi_{n}, \psi_{m}^{*}\right\}=\delta_{n+m, 0}
$$

We want the Grassmannian $G r(H)$ to consist of subspaces $W$ such that the inner product of the states corresponding to $H_{+}$and $W$ is well-defined. Recall that

$$
\langle 0 \mid W\rangle=\operatorname{det}\left\langle w_{i}, z^{j}\right\rangle
$$

in terms of the one-particle inner product $\langle\cdot, \cdot\rangle$, so $W$ and $H_{+}$must be comparable in size. This will certainly be the case if $W$ is transverse to $H_{-}$. By this we mean the following. Let $w$ be the map $H_{+} \rightarrow W$ that sends the basis elements $z^{i}$ of $H_{+}$to the basis elements $w_{i}$ of W

$$
z^{i} \rightarrow w_{i}=\sum_{j}\left(w_{+}\right)_{i j} z^{j}+\sum_{k}\left(w_{-}\right)_{i k} z^{-k}
$$

This defines for us the projections $w_{+}$and $w_{-}$. We now have

$$
\langle 0 \mid W\rangle=\operatorname{det} w_{+}
$$

We will require that $w_{+}$is invertible. In this case $W$ can be seen as the graph of the map $A=w_{+}^{-1} w_{-}$, and we can choose a basis

$$
w_{i}=z^{i}+\sum_{j=1}^{\infty} A_{i j} z^{-j}
$$

Clearly the case $A=0$ corresponds to $W=H_{+}$. In terms of matrix $A_{i j}$ the state $|W\rangle$ can be written as the Bogoliubov transform

$$
|W\rangle=h|0\rangle, \quad h=\exp \sum_{i, j} A_{i j} \psi_{i} \psi_{j}^{*}
$$

with $h$ an element of a subgroup of $G L(\infty, \mathbf{C})$ that has a natural action on the Grassmannian $\operatorname{Gr}(H)$.

There is a smaller subgroup $\Gamma_{+} \subset G L(\infty, \mathbf{C})$ defined by multiplication with a function $g(z)$ that is holomorphic on the unit disk $D_{0}$. A map $g(z) \in \Gamma_{+}$simply multiplies every element $w \in W$. With respect to the polarization $H_{+} \oplus H_{-}$of $H$ it has a decomposition of the form

$$
g=\left(\begin{array}{ll}
a & b \\
0 & c
\end{array}\right)
$$


We can now consider the orbit $\Gamma_{+} \cdot W$ of a subspace $W \in G r(H)$ under the action of $\Gamma_{+}$. If we parametrize $g(z)$ as

$$
g(t, z)=\exp \sum_{n=1}^{\infty} t_{n} z^{n}
$$

the evolution operator $U(t)$ that acts in the fermion Fock space is defined by

$$
U(t)|W\rangle=|g W\rangle
$$

Multiplication by $z^{n}$ simply shifts $z^{k} \rightarrow z^{k+n}$, so $U(t)$ is realized in terms of the fermion fields as

$$
U(t)=\exp \sum_{n=1}^{\infty} t_{n} J_{n} .
$$

Here $J_{n}$ are the modes of the fermion current $J(z)=\psi \psi^{*}(z)$, with the fundamental property $\left[J_{n}, \psi_{k}\right]=\psi_{k+n}$. We can write $U(t)$ as

$$
U(t)=\exp \oint \frac{d z}{2 \pi i} \log g(z) \cdot J(z)
$$

We are now finally in a position to explain how all this is related to the Lax pair formulation of the integrable hierarchies that we considered in the previous subsections. The subspace $W$ has, by definition, a unique element $\eta=w_{0}$ satisfying

$$
\eta(z)=1+\sum_{i=1}^{\infty} a_{i} z^{-i}
$$

It can be described formally as the intersection

$$
\eta(z)=W \cap\left(1+H_{-}\right)
$$

Similarly the transform $g^{-1} W$ contains an element with that property

$$
\eta(t, z)=g^{-1} W \cap\left(1+H_{-}\right) .
$$

Recall here that $g$ is parametrized as in (3.43). But this last equation implies directly that

$$
\psi(t, z)=g(t, z) \cdot \eta(t, z) \in W
$$

Since this is true for all times $t_{n}$ and $W$ is a linear space, we have the property that all (multiple) derivatives of $\psi$ still lie in $W$. In particular we have

$$
g^{-1} \frac{\partial \psi}{\partial t_{n}}=z^{n}+\ldots \in g^{-1} W
$$


and

$$
g^{-1} \frac{\partial^{k} \psi}{\partial x^{k}}=z^{k}+\ldots \in g^{-1} W
$$

Since $g^{-1} W$ has a unique basis of the form (3.40), this implies that $\psi$ satisfies an equation

$$
\frac{\partial \psi}{\partial t_{n}}=H_{n} \psi
$$

where $H_{n}$ is an $n^{t h}$ order differential operator in $x$. By construction the $H_{n}$ commute, and thus we have found a solution to the KP hierarchy. The reduction to KdV comes about when $W$ satisfies the extra constraint

$$
z^{p} \cdot W \subset W
$$

In that case one finds the additional relation

$$
L \psi=z^{p} \psi
$$

which defines the differential operator $L=H_{p}$.

\section{4. $\tau$-FUNCTIONS AND DETERMINANTS}

The tau-function $\tau(t)$ associated to a point $W$ in the Grassmannian is now defined as

$$
\tau(t)=\frac{\operatorname{det}\left(g^{-1} w\right)_{+}}{g^{-1} \operatorname{det} w_{+}}
$$

with $g$ parametrized as in (3.43), or in our notation (3.40)-(3.42)

$$
\tau(t)=\operatorname{det}\left(1+a^{-1} b A\right)
$$

In terms of the fermion Fock space we have the representation

$$
\tau(t)=\frac{\langle 0|U(t)| W\rangle}{g^{-1}\langle 0 \mid W\rangle}
$$

Since the state

$$
\langle t|=\langle 0| U(t)
$$

can be considered as a coherent state in the Hilbert space, the $\tau$-function can alternatively be regarded as the wave-function of the state $|W\rangle$. 
The bosonization formulas

$$
J=\partial \phi, \quad \psi=e^{\phi}, \quad \psi^{*}=e^{-\phi}
$$

lead us to consider the operators

$$
\gamma_{+}(z)=\exp \sum_{n=1}^{\infty} \frac{1}{n} z^{-n} \frac{\partial}{\partial t_{n}}
$$

Insertion of the fermi field $\psi(z)$ corresponds to action on $\tau(t)$ with the differential operator

$$
\gamma(z) \equiv \gamma_{+}(z) \cdot g(z)
$$

together with an increase in fermi number of one unit. If we denote the $N$-particle ground state as

$$
|N\rangle=z^{N} \wedge z^{N+1} \wedge z^{N+2} \wedge \ldots
$$

then we evidently have

$$
\gamma\left(z_{1}\right) \cdots \gamma\left(z_{N}\right) \cdot \tau(0)=\left\langle N\left|\psi\left(z_{1}\right) \cdots \psi\left(z_{N}\right)\right| W\right\rangle
$$

where the notation on the LHS indicates that we first take derivatives and then put the arguments $t_{n}=0$ in the $\tau$-function. This implies in particular that

$$
\gamma_{+}\left(z_{1}\right) \cdots \gamma_{+}\left(z_{N}\right) \cdot \tau(0)=\Delta(z)^{-1}\left\langle N\left|\psi\left(z_{1}\right) \cdots \psi\left(z_{N}\right)\right| W\right\rangle
$$

with

$$
\Delta(z)=\left\langle N\left|\psi\left(z_{1}\right) \cdots \psi\left(z_{N}\right)\right| 0\right\rangle=\prod_{i>j}\left(z_{i}-z_{j}\right)=\operatorname{det} z_{i}^{j-1},
$$

the Vandermonde determinant. In fact, (3.65) can be easily evaluated, since only the first $N$ one-particle states $w_{1}, \ldots, w_{N}$ contribute to give

$$
\left\langle N\left|\psi\left(z_{1}\right) \cdots \psi\left(z_{N}\right)\right| W\right\rangle=\operatorname{det}\left(w_{j-1}\left(z_{i}\right)\right) \equiv \Delta(w ; z)
$$

Here we introduced the 'generalized Vandermonde' determinant $\Delta(w ; z)$ for an arbitrary basis $\left\{w_{k}(z)\right\}$. Note that in this language the Baker-Akhiezer function is recovered as the fermion one-point function

$$
\psi(t, z)=\gamma(z) \log \tau(t)
$$


By expanding $\psi$ in powers of $z^{-1}$ we find as a corollary that our definition of $\tau$ coincides with the one given in $\S 3.2$.

These fermionic correlation functions occur naturally if we consider special elements $g(z) \in \Gamma_{+}$such that $g^{-1}$ is a $N^{t h}$ order polynomial with zeroes $z_{i}$ outside $D_{0}$

$$
g(z)^{-1}=\prod_{i=1}^{N}\left(1-z / z_{i}\right) .
$$

We can regard $g^{-1}$ to be the characteristic polynomial

$$
g(z)^{-1}=\operatorname{det}\left(1-z \cdot Z^{-1}\right)
$$

where $Z$ is an Hermitian matrix with eigenvalues $z_{1}, \ldots, z_{N}$. This corresponds to the choice of parameters $t_{n}$ as

$$
t_{n}=\frac{1}{n} \sum_{i=1}^{N} z_{i}^{-n}=\frac{1}{n} \operatorname{Tr} Z^{-n},
$$

also known as 'Miwa's coordinates' 47. We will write the tau-function in this parametrization as

$$
\tau(t)=\tau(Z)
$$

For this particular choice of $g$ we find immediately with (3.46) that

$$
\tau(Z)=\gamma_{+}\left(z_{1}\right) \cdots \gamma_{+}\left(z_{N}\right) \cdot \tau(0)
$$

which we already evaluated to be ratio

$$
\tau(Z)=\Delta(w ; z) / \Delta(z)
$$

This is our main results of this section. It gives a closed expression for the $\tau$-function in the specific parametrization (3.71) once we know an explicit basis $w_{k}(z)$ for the point $W \in G r(H)$.

\section{Matrix Integrals}

We will now turn to the application of the results obtained in the previous section to the specific solutions of the KdV hierarchies that appear in theories of quantum gravity. 
In this section we will perform the 'Wick rotation' $x \rightarrow i x, t_{n} \rightarrow i t_{n}$, in order to achieve better convergence properties of many of our quantities. This change is of course completely irrelevant on the level of asymptotic expansions. Note that the derivative $D=-i \partial / \partial x$ is now an Hermitian operator. We will assume that the Lax operator $L$ has (possibly complex) coefficients such that it is Hermitian too. It consequently has real eigenvalues $z^{p}$.

\subsection{The STRing EQUATion And Airy FunCtions}

We can think of the many different solutions to the KdV hierarchies, such as the famous soliton solutions, as being related to different initial conditions $L(0)$ for the Lax operator $L(t)$. For the solutions that appeared in $\S 2$ these initial conditions can be found as follows. Recall that in these cases the $\tau$-function has a very concrete interpretation: it is given by the string partition function of the $(p, 1)$ minimal topological model. Stated otherwise, the logarithm of $\tau$ is a generating functional for all correlation functions on connected surfaces of arbitrary genus $g$, and consequently has an expansion

$$
\log \tau=\sum_{g=0}^{\infty}\left\langle\exp \sum_{n} t_{n} \mathcal{O}_{n}\right\rangle_{g}
$$

If we think of the variables $t_{n}$ as describing a background, then $\log \tau$ becomes indeed the 'string free energy'

$$
\log \tau=F=\langle 1\rangle_{t}
$$

where the subscript $\langle\cdot\rangle_{t}$ simply indicates the insertion of the ' $\exp t_{n} \mathcal{O}_{n}$ ' term and a summation

over all genera is understood. In this notation equations $(3.22)-(3.23)$ relate the residues with special two-point functions

$$
\operatorname{res} K=-\left\langle\mathcal{O}_{1}\right\rangle_{t} \quad \operatorname{res} L^{n / p}=\left\langle\mathcal{O}_{1} \mathcal{O}_{n}\right\rangle_{t}
$$

We now want to determine the initial condition $L(0)$, where we have put all times $t_{n}$ to zero for $n>1$. Putting the coupling constant $t_{1}=x$ to a non-zero value and expanding $\log \tau$ up to first order in the other coupling constants, we find for $i=1, \ldots, p-1$ with (2.44)

$$
\operatorname{res} L^{i / p}=p \cdot \delta_{i, p-1} \text {. }
$$

(The factor $p$ that appears here can be absorbed in the string coupling constant, and we will drop it in the consequent.) This translates in the following initial value for the differential operator $L$

$$
L(0)=D^{p}+x
$$


That is, only a linear potential $u_{0}(x)=x$ and no higher derivative terms appear. The equation

$$
u_{i}(x)=x \cdot \delta_{i, 0}
$$

or, equivalently,

$$
[D, L]=1
$$

is known as the string equation for the $(p, 1)$ model, in the notation of the introduction.f It is this extremely trivial form of the string equation that leads to the complete solvability. Indeed, we will now be able to explicitly solve for the $\tau$-function, because in this case the Baker-Akhiezer function

$$
L \psi(x, z)=z^{p} \psi(x, z)
$$

is simply given by the generalized Airy function

$$
\psi(x, z)=c(z) \cdot \int d y \exp i\left[\left(x-z^{p}\right) y+\frac{y^{p+1}}{p+1}\right]
$$

This should be contrasted with the much more complicates solutions for an arbitrary $(p, q)$ model [48. The coefficient $c(z)$ is fixed by requiring the asymptotic expansion (3.28)

$$
\psi(x, z)=e^{i x z}\left(1+\sum_{i=1}^{\infty} a_{i}(x) z^{-i}\right)
$$

The normalization can be chosen as

$$
c(z)=e^{\frac{i p}{p+1} z^{p+1}} \sqrt{z^{p-1}}
$$

The asymptotic expansion is now verified by shifting $y \rightarrow y+z$ in the integral (4.9), so that one obtains the representation

$$
\psi(x, z)=e^{i x z} \sqrt{z^{p-1}} \int d y \cdot e^{i x y+i S(y, z)}
$$

with

$$
S(y, z)=\frac{1}{p+1}\left[(y+z)^{p+1}\right]_{\geq 2} .
$$

Here the subscript ' $\geq 2$ ' implies that we only keep terms in $y$ of second order or higher. By using the stationary phase approximation to such integrals one then easily deduces the expansion (4.10).

${ }^{*}$ For general $(p, q)$ it reads $\left[H_{q}, L\right]=1$ [7], which in the case $(p, q)=(2,3)$ gives the celebrated Painlevé equation $u^{2}+\frac{1}{6} u^{\prime \prime}=x$ of 四. 
We now wish to determine the function $\tau(Z)$ for this special initial value condition

$$
L=D^{p}+x .
$$

According to the general discussion of section 3, the Baker-Akhiezer function $\psi(t, z)$ is an element of $W$ for all values of $t$. This is in particular true if we restrict to the parameter $t_{1}=x$. So, all the functions $v_{k}(z)$ that appear in the Taylor expansion

$$
\psi(x, z)=\sum_{k=0}^{\infty} v_{k}(z) \frac{x^{k}}{k !} .
$$

are elements of $W$ :

$$
v_{k}(z) \in W .
$$

Furthermore, the functions $v_{k}(z)$ form a basis, since they are given by

$$
v_{k}(z)=\sqrt{z^{p-1}} \int d y \cdot(y+z)^{k} \cdot e^{i S(y, z)},
$$

and thus have to property

$$
v_{k}(z)=z^{k}\left(1+O\left(z^{-1}\right)\right) .
$$

They therefore define an element of the Fock space by

$$
|W\rangle=v_{0} \wedge v_{1} \wedge v_{2} \wedge \ldots
$$

and the $\tau$-function is, according to the main result of $\S 3$, simply obtained as the following ratio of determinants

$$
\tau(Z)=\Delta(v ; z) / \Delta(z) .
$$

\subsection{More General models: the Small Phase SPaCe}

One can slightly generalize the previous case by considering an operator of the form

$$
L=W(D)+x,
$$

with $W$ an arbitrary polynomial of order $p$ with constant coefficients

$$
W(y)=y^{p}+g_{p-2} y^{p-2}+\ldots+g_{0} .
$$


If we introduce a potential $V$ by the relation

$$
W(y)=V^{\prime}(y)
$$

and a function $\zeta(z)$ through

$$
V^{\prime}(\zeta)=z^{p}, \quad \zeta=z+O\left(z^{-1}\right)
$$

the Baker-Akhiezer function reads in this case

$$
\psi(x, z)=e^{i x \zeta} \sqrt{V^{\prime \prime}(\zeta)} \int d y \cdot e^{i x y+i S(y, \zeta)}
$$

with $S$ defined as

$$
S(y, \zeta)=[V(y+\zeta)]_{\geq 2}=V(y+\zeta)-V(\zeta)-y V^{\prime}(\zeta)
$$

In this case the moments $v_{k}(z)$ are given by

$$
v_{k}(z)=\sqrt{V^{\prime \prime}(\zeta)} \int d y \cdot(y+\zeta)^{k} \cdot e^{i S(y, \zeta)}
$$

This case is actually of great interest, since an operator of the form (4.21) is naturally obtained if we let the operator $L=D^{p}+x$ evolve in the 'primary' times $t_{1}, \ldots, t_{p-1}$. The subspace parametrized by these $t_{i}$ 's is known as the 'small phase space' [15. In intersection theory the restriction to the small phase space implies that we only consider the top Chern class of the vector bundle $V$, not the Mumford-Morita-Miller classes $\sigma_{n}$. In the corresponding quantum field theory the primary operators $\mathcal{O}_{1}, \ldots, \mathcal{O}_{p-1}$ are the physical fields of a $(p, 1)$ topological minimal model. The small phase space describes the moduli space of topological field theories that can be reached by perturbation of the minimal models. When we consider $\tau(t)$ as a function on the small phase space, it receives furthermore, by charge conservation, only contributions at genus zero.

In the context of integrable hierarchies of $\mathrm{KdV}$ type, these special properties of the first $p-1$ flows are reflected in the fact that the corresponding Hamiltonians $H_{i}=L_{+}^{1 / p}$ do not depend on $x$. Therefore the dispersionless, or spherical, approximation is exact, and we have the classical equations

$$
\frac{\partial L}{\partial t_{i}}=\left\{H_{i}, L\right\}=\left\{L_{+}^{i / p}, x\right\}=\frac{\partial L_{+}^{i / p}}{\partial y}
$$


with $y$ the classical momentum corresponding to $D$. These equations fully determine the $t$-dependence of $L$. The initial value

$$
W(y)=y^{p}
$$

evolves to a general potential of type (4.22), where the coefficients $g_{i}(t)$ are given implicitly by the relation

$$
i(p-i) t_{p-i}=\operatorname{res} L^{i / p}=\oint \frac{d y}{2 \pi i} L(y)^{i / p}, \quad(i=1, \ldots, p-1) .
$$

This equation follows immediately from the results (4.3) and (2.44).

\subsection{MATRIX INTEGRALS}

We will now show following Kontsevich [22] how the previous results are related to matrix integrals. First recall the wonderful result of Harish-Chandra [50], Mehta [51], Itzykson and Zuber [52], for the following integral over the unitary group $U(N)$

$$
\int d U \exp i \operatorname{Tr}\left[U X U^{\dagger} Y\right]=c \cdot \frac{\operatorname{det} e^{i x_{i} y_{j}}}{\Delta(x) \Delta(y)}
$$

with $d U$ the Haar measure and $x_{i}, y_{i}$ the eigenvalues of the Hermitian matrices $X$ and $Y$. This result can be obtained in many different ways, perhaps most elegantly as an application of the Duistermaat-Heckman localization formula [53].

As a special application of the above equation consider the matrix Fourier transform, i.e., the integral over a hermitian matrix $Y$ in a external field $X$, both $N \times N$ matrices, of the form

$$
\tau(X)=\int d Y \exp i \operatorname{Tr}[X Y+V(Y)]
$$

By conjugation invariance, this is only a function of the eigenvalues $x_{1}, \ldots, x_{N}$. We can use (4.31) to integrate out the angular variables $U$ in the decomposition

$$
Y=U \cdot \operatorname{diag}\left(y_{1}, \ldots, y_{N}\right) \cdot U^{\dagger}
$$

which also introduces a Jacobian

$$
d Y=\Delta(y)^{2} \cdot d U \cdot[d y]
$$


This leaves us with an integral over the eigenvalues $y_{i}$ of the form

$$
\tau(X)=\int[d y] \Delta(y) \Delta(x)^{-1} \exp \sum_{j} i\left[x_{j} y_{j}+V\left(y_{j}\right)\right]
$$

Now the Vandermonde determinant $\Delta(y)$ is a sum of terms of the form

$$
\pm y_{1}^{i_{1}} \cdots y_{N}^{i_{N}}
$$

and for each of these terms the integral $\tau(X)$ factorizes in separate integrals over the individual eigenvalues $y_{i}$. If we introduce the function

$$
w(x)=\int d y \cdot e^{i x y+i V(y)}
$$

and its derivatives

$$
w_{k}(x)=\int d y \cdot y^{k} \cdot e^{i x y+i V(y)},
$$

the contribution of (4.36) is simply

$$
\pm w_{i_{1}}\left(x_{1}\right) \cdots w_{i_{N}}\left(x_{N}\right)
$$

So we can evaluate $\tau(X)$ straightforwardly as

$$
\tau(X)=\operatorname{det}\left(w_{j-1}\left(x_{i}\right)\right) / \Delta(x)=\Delta(w ; x) / \Delta(x)
$$

in the notation of the previous section. This very much suggests that $\tau(X)$ is a $\tau$-function for the KP hierarchy with one-particle wave-functions $w_{0}, w_{1}, \ldots$ This will be the case if these functions have appropriate asymptotic expansions, and so depends on the choice of potential $V$ and the normalization of the integral.

We first wish to apply this result using the functions $v_{k}(z)$ that appeared in $\S 4.1$. Recall that these where the derivatives (at $x=0$ ) of the Baker-Akhiezer function of the operator $L=D^{p}+x$. In this case one simply puts

$$
V(Y)=\frac{1}{p+1} Y^{p+1}, \quad X=-Z^{p}
$$

and

$$
\tau(Z)=c(Z) \cdot \int d Y \exp i \operatorname{Tr}\left[-Z^{p} \cdot Y+\frac{Y^{p+1}}{p+1}\right]
$$


with a proper choice of normalization $c(Z)$. This constant is basically the product of the individual constants $c\left(z_{i}\right)$ of (4.11), together with a correction replacing the Vandermonde determinant $\Delta\left(z^{p}\right)$ by $\Delta(z)$

$$
c(Z)=\operatorname{det} Z^{\frac{p-1}{2}} \cdot e^{\frac{i p}{p+1} \operatorname{Tr} Z^{p+1}} \frac{\Delta\left(z^{p}\right)}{\Delta(z)} .
$$

With all these ingredients the integral (4.42) reproduces indeed our $\tau$-function

$$
\tau(Z)=\Delta(v ; z) / \Delta(z)
$$

The integral can be written in yet another fashion. Let $S(Y, Z)$ be given as in (4.13)

$$
S(Y, Z)=\frac{1}{p+1} \operatorname{Tr}\left[(Y+Z)^{p+1}\right]_{\geq 2}
$$

and let $S_{2}(Y, Z)$ denote the part of the function $S(Y, Z)$ of second order in $Y$

$$
S_{2}(Y, Z)=\sum_{k=0}^{p-1} \operatorname{Tr}\left[Y Z^{k} Y Z^{p-1-k}\right]
$$

One easily evaluates the Gaussian integral

$$
\rho(Z) \equiv \int d Y e^{i S_{2}(Y, Z)}=\prod_{i, j} \sqrt{\frac{z_{i}-z_{j}}{z_{i}^{p}-z_{j}^{p}}}=\operatorname{det} Z^{\frac{1-p}{2}} \frac{\Delta(z)}{\Delta\left(z^{p}\right)}
$$

This can be used to rewrite $\tau(Z)$ as

$$
\tau(Z)=\rho(Z)^{-1} \int d Y e^{i S(Y, Z)}
$$

This matrix integral has been suggested as the relevant one for the $(p, 1)$ model by several authors, see e.g. 22, 49, 27]. Here we have shown that it follows naturally from the Lax pair formulation of the string equation, by which the general $(p, q)$ model is characterized.

The integral (4.48) has an obvious asymptotic expansion in matrix Feynman diagrams. In this way we recover the cell decomposition used by Kontsevich to derive his results for $p=2$. This elegant geometrical interpretation is less clear for higher values of $p$. First of all one must include higher order vertices (up to order $p+1$ ), which implies the consideration of cells in $\mathcal{M}_{g, s}$ of non-zero codimension. Secondly, the Feynman rules associated to these 
vertices become $Z$-dependent, and generally quite complicated. It would be very interesting if this prescription could nevertheless be directly related to the intersection formulas of $\S 2.4$.

As is also observed in [22, 27], the formula (4.48) makes sense for a much bigger class of potentials. We can consider the general case

$$
\tau(Z)=c(Z) \cdot \int d Y \exp i \operatorname{Tr}[-W(Z) \cdot Y+V(Y)]
$$

If $V(Y)$ has a well-defined Taylor expansion around $Y=Z$, we can put

$$
W(Y)=V^{\prime}(Y)
$$

After a shift in the integration variable $Y$, the matrix integral $\tau(Z)$ can be written in the form (4.48), if we define the action to be

$$
S(Y, Z)=\operatorname{Tr}\left[V(Y+Z)-V(Z)-Y \cdot V^{\prime}(Z)\right]
$$

and $S_{2}(Y, Z)$ similarly as in (4.46) as the quadratic part of $S$. One easily calculates

$$
\rho(Z)=\left(\operatorname{det} V^{\prime \prime}(Z)\right)^{-1 / 2} \frac{\Delta(z)}{\Delta\left(V^{\prime}(z)\right)}
$$

and the matrix integral (4.48) becomes again a ration of determinants

$$
\tau(Z)=\Delta(v ; z) / \Delta(z)
$$

In this more general case the functions $v_{k}(z)$ are given by

$$
v_{k}(z)=\sqrt{V^{\prime \prime}(z)} \int d y \cdot(y+z)^{k} \cdot e^{i S(y, z)}
$$

and, again under some mild restriction on the potential $V$, this produces a solution of the KP hierarchy.

One might wonder if all these solutions are completely independent. This brings us to the following point. The most general matrix integral that naturally leads to a $\tau$-function of the KP hierarchy depends both on the choice of potential $V$ and the external field $Z$. This might be a redundant parametrization, in the sense that a particular variation $\delta V$ corresponds to a flow in the times $t_{n}$ encoded by $Z$. We meet a simple example of this phenomenon if $V(Y)$ 
is a polynomial of order $p+1$. In this case the functions $v_{k}$ that we constructed above are identical to the ones obtained in $\S 4.2$, after we perform a reparametrization

$$
z \rightarrow \zeta(z), \quad \zeta^{p}=W(z)
$$

Recall that we considered at that point the $\tau$-function associated to the Lax operator $L=$ $W(D)+x$. A Lax operator of that form could be obtained, if we start with $W(Y)=Y^{p}$ and flow in the primary times $t_{1}, \ldots, t_{p-1}$. Indeed, as we showed, in that case one ends up with a general polynomial $W(Y)$ of order $p$ whose coefficients are determined through the relation

$$
t_{p-i}=\frac{1}{i(p-i)} \operatorname{res} W^{i / p}, \quad i=1, \ldots, p-1
$$

We now see that the correct identification of the KP times $t_{n}$ is given by

$$
t_{n}=\frac{1}{n} \operatorname{Tr} W(Z)^{-n / p}
$$

which just expresses the reparametrization (4.55) of the coordinate $z$. Summarizing, the change in potential can be absorbed in the redefinition in the coupling coefficients (4.57), together with the extra term (4.56) for the primary couplings. Of course, it is possible to consider more general flows, where the order of the potential changes. This cannot be absorbed in a shift of the $\mathrm{KdV}$ times, and in this way one can interpolate between different values of $p$.

As a final application, we can consider the limit $p \rightarrow-1$. In that case we find

$$
W(Y)=Y^{-1}, \quad V(Y)=\log Y
$$

One easily verifies that the Kontsevich matrix model now becomes identical to Penner's model that computes the Euler characteristic of moduli space, in accordance with our considerations of $\S 2.4$

\subsection{The Virasoro CONSTRAints}

We will make here a few brief comments on the occurrence of Virasoro algebras (and more general $W$-algebras) in the context of these integrable hierarchies. We already mentioned this characterization of the tau-function in the introduction and we will see in $\S 7$ that it occurs naturally in the context of topological string theory. There exists by now a quite extensive literature on this subject, see e.g. our selection [54], and we will be very brief at this point. 
Let us start with a few definitions. In the free fermion theory there is a holomorphic stress tensor

$$
T(z)=\frac{1}{2}\left(\partial_{z} \psi^{*} \psi-\psi^{*} \partial_{z} \psi\right)=\frac{1}{2} J^{2}
$$

which describes the behaviour of the quantum theory under reparametrizations $z \rightarrow w(z)$ of the unit circle. Its modes $L_{n}$, defined by

$$
T(z)=\sum_{n} L_{n} z^{-n-2}
$$

satisfy the $c=1$ Virasoro algebra

$$
\left[L_{n}, L_{m}\right]=(n-m) L_{n+m}+\frac{1}{12} n\left(n^{2}-1\right) \delta_{n+m, 0}
$$

and are the infinitesimal generators of $\operatorname{Diff}\left(S^{1}\right)$. They can be represented as differential operators acting on the $\tau$-function through the relation

$$
L_{n_{1}} \cdots L_{n_{k}} \cdot \tau(t)=\left\langle t\left|L_{n_{1}} \cdots L_{n_{k}}\right| W\right\rangle \text {. }
$$

This gives, with $n>0$, the explicit realizations

$$
\begin{aligned}
L_{-n} & =\sum_{k=n+1}^{\infty} k t_{k} \frac{\partial}{\partial t_{k-n}}+\frac{1}{2} \sum_{i+j=n} i j t_{i} t_{j}, \\
L_{0} & =\sum_{k=1}^{\infty} k t_{k} \frac{\partial}{\partial t_{k}} \\
L_{n} & =\sum_{k=1}^{\infty} k t_{k} \frac{\partial}{\partial t_{k+n}}+\frac{1}{2} \sum_{i+j=n} \frac{\partial^{2}}{\partial t_{i} \partial t_{j}} .
\end{aligned}
$$

In a $\tau$-function of the $p^{t h} \mathrm{KdV}$ hierarchy all $t_{k}$ 's with $k \equiv 0(\bmod p)$ can consistently be put to zero, and only the modes of the form $L_{q \cdot p}$ act non-trivially.

In the previous sections we have constructed a special solution of the $p^{\text {th }} \mathrm{KdV}$ hierarchy, that turns out to have remarkably simple properties with respect to the Virasoro generators. The results can be formulated as follows. Let us introduce the redefined operators $L_{n}^{\prime}$ by

$$
p \cdot L_{n}^{\prime}=L_{n \cdot p}-\frac{\partial}{\partial t_{1+(n+1) p}}+\delta_{n, 0} \frac{p^{2}-1}{24}, \quad n \geq-1 .
$$

One easily verifies that they satisfy the algebra

$$
\left[L_{n}^{\prime}, L_{m}^{\prime}\right]=(n-m) L_{n+m}^{\prime}
$$


The $\tau$-function of the $(p, 1)$ model can now be shown to obey the equations

$$
L_{n}^{\prime} \cdot \tau=0, \quad n \geq-1
$$

A derivation of this result is given in [24]. These equations have a straightforward interpretation as recursion relations for correlation functions, as we will see explicitly for the case $p=2$ in $\S 7$.

There exists actually a much bigger algebra of currents bilinear in the fermions - the so-called $W_{1+\infty}$ algebra [56], generated by currents of the form

$$
W(z)=\partial_{z}^{i} \psi^{*} \partial_{z}^{j} \psi
$$

The modes of this fields can also be realized as differential operators, and can be considered as the infinitesimal generators of the group $G L(\infty, \mathbf{C})$ that acts naturally on Sato's Grassmannian. The $\tau$-function of the $(p, 1)$ model can be shown to satisfy the more general constraints

$$
W_{n}^{(s)} \cdot \tau=0, \quad s=2, \ldots, p, n \geq 1-s,
$$

where $W_{n}^{(s)}$ are the modes of a spin $s$ generator $W^{(s)}(z)$. See e.g. [55, 56] for more details. The fields $W^{(s)}$ generate a so-called $W$-algebra associated to the Lie group $S L(p)$.

These constraints can actually be found directly in the matrix model representation of the $\tau$-function [57]. A very elegant way [21, 58, 59] proceeds as follows. Recall that for the $(p, 1)$ models the $\tau$-function was, up to normalization, given by the generalized matrix Airy function

$$
A(X)=\int d Y \cdot \exp i \operatorname{Tr}\left[X Y+\frac{Y^{p+1}}{p+1}\right] .
$$

This function satisfies the 'matrix Airy equation'

$$
\left(\frac{\partial^{p}}{\partial X^{p}}+X\right) A=0
$$

a reflection in matrix terms of the 'string equation' $L=D^{p}+x$. When this equation is written out in terms of the coordinates $t_{n}$, its gives rise to a set of equations of the form

$$
Q \cdot \tau=0
$$

where $Q$ is a differential operators of order $p$ or less. The claim is, that these equations reproduces exactly the Virasoro and $W$-constraints. 


\section{Topological Field Theory}

We now shift our perspective completely and start our discussion of topological field theories [60, 61]. The models we consider in this and the next section can be considered as 'matter theories' and only describe the primary fields $\mathcal{O}_{1}, \ldots, \mathcal{O}_{p-1}$ that featured in our previous models. Consequently, we will only recover the KdV equations on the small phase space. In order to extend our discussion to the full integrable hierarchies these models have to be coupled to (topological) gravity - a subject that we will postpone until $\S 7$, and then only discuss marginally.

\subsection{The Stress-energy tensor}

Let us start with some remarks of a rather general nature. For other introductions see e.g. 62]- 64]. Given a compact manifold $M$ of dimension $D$ and a quantum field theory with some set of fundamental fields $\phi(x)$, one can consider the vacuum amplitude or partition function

$$
Z(M)=\int[d \phi] e^{-S[\phi]}
$$

Here we formally defined $Z(M)$ by the path-integral over all field configurations on $M$ weighted with some action $S$. In general the partition function $Z(M)$ will depend on many geometrical data. For instance, in almost all quantum field theories we need a Riemannian structure on $M$, i.e., a metric $g_{\mu \nu}(x)$. This metric enters both in the definition of the action $S$ and in the definition of the measure $[d \phi]$. Other possible ingredients might be an orientation and, if fermions are involved, a choice of spin structure, or in the case of gauge fields a choice of fiber bundle.

Although through its definition the quantum field theory can seem to depend on all information encoded in the metric $g_{\mu \nu}$, it might be the case that the actual amplitudes have some invariance under particular changes $\delta g_{\mu \nu}$ in the metric. An example is the invariance under reparametrizations

$$
\delta g_{\mu \nu}=D_{\mu} \epsilon_{\nu}+D_{\nu} \epsilon_{\mu}
$$

As is well-know, this leads to the conservation law

$$
D^{\mu} T_{\mu \nu}=0
$$

for the expectation value of the stress-energy tensor

$$
T_{\mu \nu}=\frac{1}{\sqrt{g}} \frac{\delta S}{\delta g^{\mu \nu}}
$$


that encodes the reaction of the quantum field theory to metric deformations. Another example of a symmetry is conformal invariance: the invariance under local rescalings of the metric

$$
\delta g_{\mu \nu}=\epsilon \cdot g_{\mu \nu}
$$

with $\epsilon(x)$ an arbitrary function. This implies that the trace of $T_{\mu \nu}$ vanishes

$$
T_{\mu}^{\mu}=0
$$

Quantum field theories with this property are known as conformal field theories.

A quantum field theory will be called topological, when it is invariant under arbitrary smooth deformation of the metric:

$$
\delta g_{\mu \nu}=\epsilon_{\mu \nu}
$$

In this fashion only the topology of the manifold $M$ will matter, and the partition function $Z(M)$ will be a topological invariant. In this case the stress tensor will have to vanish completely

$$
T_{\mu \nu}=0 .
$$

This discussion can be generalized from vacuum amplitudes to physical correlation functions. In a generic quantum field theory, the correlation functions depend on the position of the operators. But in a topological field theory the physical correlation functions of local operators are just numbers,

$$
\left\langle\phi_{i_{1}}\left(x_{1}\right) \cdots \phi_{i_{s}}\left(x_{s}\right)\right\rangle_{M} \equiv \int[d \phi] \phi_{i_{1}}\left(x_{1}\right) \cdots \phi_{i_{s}}\left(x_{s}\right) \cdot e^{-S}=\text { constant }
$$

This is again a manifestation of the metric-independence of physical observables, and a consequence of the vanishing of the stress-energy tensor, now within correlation functions

$$
\left\langle T_{\mu \nu}(x) \phi_{i_{1}}\left(x_{1}\right) \cdots \phi_{i_{s}}\left(x_{s}\right)\right\rangle_{M}=0
$$

In $§ 5.4$ we will see how these conditions can be realized in practice.

\subsection{FaCtorization AND Atiyah's AXIOMS}

We will at this point not be interested in actually evaluating the path-integral for a given quantum field theory. We will rather, following Atiyah [65], deduce from the above definition certain properties of partition functions, and more generally correlation functions, that can be lifted to the status of 'axioms.' The most important property is the concept of factorization. 
Let us in our imagination cut the manifold $M$ along a codimension one subspace $B$, so that $M$ splits into two parts $M_{1}$ and $M_{2}$. In this case we can do the path-integral in two steps. Let us first fix the values of the fields $\phi(x)$ to some given configuration $\phi^{\prime}$ on $B$, and do the separate integrals over $M_{1}$ and $M_{2}$ with these boundary conditions. If we write

$$
\Psi_{M_{i}}\left(\phi^{\prime}\right)=\int_{\left.\phi\right|_{B}=\phi^{\prime}}[d \phi] e^{-S[\phi]}
$$

for the path-integral over the component $M_{i}$ with fixed values $\phi^{\prime}$ at $B$, it is clear that we will have

$$
Z(M)=\int\left[d \phi^{\prime}\right] \Psi_{M_{1}}\left(\phi^{\prime}\right) \cdot \Psi_{M_{2}}\left(\phi^{\prime}\right)
$$

To make this more precise we have to consider general space-times $M$ with boundaries. To each connected component $B$ of the boundary $\partial M$ of $M$ a quantum field theory will associate a Hilbert space of states $\mathcal{H}_{B}$

$$
B \rightarrow \mathcal{H}_{B}
$$

In explicit examples this Hilbert space will be constructed by considering canonical quantization on the cylinder $B \times \mathbf{R}$, where $B$ is considered space-like and $\mathbf{R}$ the time direction. States $|\Psi\rangle \in \mathcal{H}_{B}$ can be represented as wave functions $\Psi(\phi)=\langle\phi \mid \Psi\rangle$ of the fundamental field variables $\phi(x)$ restricted to $B$. In our examples the vector spaces $\mathcal{H}_{B}$ will actually be finite-dimensional.

The assignment of vector spaces (5.13) should satisfy some intuitively clear axioms. If the boundary $\partial M$ has several disconnected components $B_{1}, \ldots, B_{s}$ we define

$$
\mathcal{H}_{\partial M}=\mathcal{H}_{B_{1}} \otimes \cdots \otimes \mathcal{H}_{B_{s}}
$$

Furthermore, if the boundary is empty, $\mathcal{H}$ is one-dimensional

$$
\mathcal{H}_{\emptyset}=\mathbf{C}
$$

The definition of $\mathcal{H}_{B}$ will in general depend on the orientation of $B$. If $-B$ denotes the manifold with reversed orientation, we have

$$
\mathcal{H}_{-B}=\mathcal{H}_{B}^{*}
$$

since the path-integral on the cylinder $B \times \mathbf{R}$ gives a canonical isomorphism

$$
\mathcal{H}_{B} \otimes \mathcal{H}_{-B} \rightarrow \mathbf{C}
$$


The space-time $M$ will defines a certain state in the Hilbert space associated to its own boundary $\partial M=B$

$$
|M\rangle \in \mathcal{H}_{B}
$$

where the orientation of $M$ and $B$ agree. Intuitively, the wave function $\Psi_{M}\left(\phi^{\prime}\right)$ representation of this state is defined as the path-integral over all field configurations $\phi$ on $M$ that restrict to $\phi^{\prime}$ at the boundary as in (5.11), see fig. 4.

In general we can consider the case that we give part of $\partial M$ (say $B^{\prime}$ ) an orientation that agrees with that of $M$, and the other part $(B)$ the reverse orientation, as in fig. 5 . In that case the path-integral on $M$ will define an element in $\mathcal{H}_{B}^{*} \otimes \mathcal{H}_{B^{\prime}}$, or equivalently a transition amplitude

$$
\Phi_{M}: \mathcal{H}_{B} \rightarrow \mathcal{H}_{B^{\prime}}
$$

Factorization is now the property that if we cut $M$ in two parts $M_{1}$ and $M_{2}$ along an intermediate slice $B^{\prime \prime}$, the transition amplitudes satisfy

$$
\Phi_{M}=\Phi_{M_{2}} \circ \Phi_{M_{1}}
$$

This property is illustrated in fig. 6. Since we compose linear operators, the process includes a sum over states in the intermediate Hilbert space $\mathcal{H}_{B^{\prime \prime}}$.

We should stress that this type of factorization is characteristic of 'matter theories.' Theories of gravity, that somehow include an integral over all metrics on $M$, behave characteristically differently. This is due to the fact that in quantum gravity the path-integral only becomes well-defined if one integrates over all metrics modulo diffeomorphisms. It is clear that not all diffeomorphisms of a manifold $M$ respect the decomposition $M=M_{1} \cup M_{2}$, so factorization of the path-integral is not obvious. Another important modification in theories of quantum gravity is the natural and unavoidable occurrence of singular configurations, where the topology of the manifold actually changes. In Polyakov's word, the space of metrics has a boundary of degenerate manifolds, and the path-integral naturally receives contributions of this boundary. We met this phenomenon already when we discussed the stable compactification of the moduli space of Riemann surfaces in $§ 2.1$.

\subsection{TOPOLOGICAL FIELD THEORY IN TWO DIMENSIONS}

In this subsection we will restrict our investigations to two dimensions $(D=2)$ where our manifolds $M$ are surfaces of genus $g$. Since the only connected compact one-dimensional manifold is the circle $S^{1}$, we have only one vector space to consider

$$
\mathcal{H} \equiv \mathcal{H}_{S^{1}}
$$


and for convenience we will assume it to be finite dimensional

$$
\operatorname{dim} \mathcal{H}=N<\infty
$$

The data of a two-dimensional topological field theory are now obtained by considering respectively the sphere with one, two, and three holes [66]. Let us briefly run through the argument.

(i) The disk gives rise to a particular state

$$
1 \in \mathcal{H}
$$

that we will denote as the identity, for reasons that become obvious in a moment.

(ii) The cylinder gives a bilinear map

$$
\eta: \mathcal{H} \otimes \mathcal{H} \rightarrow \mathbf{C}
$$

which we notate as

$$
\eta(a, b)=\langle a, b\rangle
$$

By factorization, this inner product $\eta$ will be non-degenerate, but not necessarily positive. It allows us to identify the 'incoming states' in $\mathcal{H}$ with the 'outgoing states' in the dual space $\mathcal{H}^{*}$. Note that here both boundary components have an orientation that is opposite to that of the cylinder, and the map $\eta$ should be sharply distinguished from the identity map (5.17).

(iii) Finally the pair of pants,- - or the sphere with three holes - corresponds, again with the appropriate choice of orientations, to a bilinear map

$$
c: \mathcal{H} \otimes \mathcal{H} \rightarrow \mathcal{H}
$$

If we introduce the notation

$$
c(a, b)=a \times b,
$$

this makes $\mathcal{H}$ into an algebra, the operator product algebra of the topological field theory. Indeed, this allow us to identify states with operators in a very simple way. It is convenient to choose an explicit basis $\left\{\phi_{0}, \ldots, \phi_{N-1}\right\}$ for $\mathcal{H}$, with $\phi_{0}=\mathbf{1}$, in terms of which we have in component notation

$$
\eta_{i j}=\left\langle\phi_{i}, \phi_{j}\right\rangle, \quad \phi_{i} \times \phi_{j}=\sum_{k} c_{i j}^{k} \phi_{k}
$$

All these data suffice to calculate any partition or correlation function, since every surface can by factorization be reduced to a collection of three-holed spheres. Of course, there are many 
inequivalent ways to factorize a surface. The final answer should however not depend on the particular choice of factorization. This concept, known as duality, gives further constraint on the data $N, \eta$ and $c$. For instance, a simple consequence of the symmetry of the 3-punctured sphere is the compatibility of the metric $\eta$ with the algebra $\mathcal{H}$

$$
\langle a \times b, c\rangle=\langle a, b \times c\rangle .
$$

If we consider the sphere with four holes, there are two inequivalent ways of factorization as illustrated in fig. 7. This leads to so-called $s$-t duality, which translates in associativity of the algebra $\mathcal{H}$

$$
(a \times b) \times c=a \times(b \times c) .
$$

It can be easily checked that no further conditions will be found when we consider more complicated surfaces.

With the concept of factorization, it is extremely easy to calculate higher genus partition and correlation functions. In fact, we can introduce an operator $H$ that creates an handle [10]. It is defined as the state associated to the torus with one puncture (see fig. 8), and has the representation

$$
H=\sum_{a \in \text { basis }}\left(\operatorname{Tr} a^{*}\right) \cdot a=\sum_{i, j} c_{i}^{i j} \phi_{j}
$$

In this fashion a genus $g$ partition function $Z_{g}$ can be written as a genus zero correlation function

$$
Z_{g}=\langle\underbrace{H \cdots H}_{g}\rangle_{0}
$$

From this expression it is immediately clear that for topological field theories, in which the factorization axiom holds, partition functions grow polynomial for large genus

$$
Z_{g} \sim c^{g}
$$

with some constant $c$. This behaviour contrasts starkly with results in two-dimensional quantum gravity and string theory, where the size of the partition function is related to the volume of moduli space and has the famous factorial growth 67

$$
Z_{g} \sim(2 g) !
$$

This already indicates to us, that the simple kind of general covariance that we are studying in this and the following section, is not the one relevant for gravity theories. It has to be appropriately modified - we will have to give up factorization - and we will return to these 
modifications in section 7. However, before we discuss all this, we will first have to explain how topological invariance can be realized in practice. Hereto we will consider again the general $D$-dimensional case.

\subsection{The $Q$-Cohomology}

It might not be obvious how all of the above structures can be realized in a quantum field theory. Indeed, one does not expect in general such a simple structure; finite dimensional Hilbert spaces are already exceptional in quantum mechanics, let alone quantum field theory. The crucial ingredient is to introduce a cohomology principle into the game.

The metric independence independence of a quantum field theory was expressed by the vanishing of the energy-momentum tensor

$$
T_{\mu \nu}=0 .
$$

In theories of quantum gravity this general covariance is achieved by making the metric $g_{\mu \nu}$ a dynamic variable and integrating over all metrics in the path-integral. The stress tensor of the matter is now balanced by the contribution of the gravity sector

$$
T_{\mu \nu}=T_{\mu \nu}^{(\text {matter })}+T_{\mu \nu}^{(\text {gravity })}=0
$$

However, the introduction of a fluctuating metric can be avoided if the quantum field theory contains a fermionic charge $Q$ that is nilpotent

$$
Q^{2}=0
$$

One can think about $Q$ as a generating a BRST-like symmetry, like in gauge theories, and define a physical Hilbert space $\mathcal{H}$ as the cohomology ring of $Q$ in the full Hilbert space $\mathcal{H}^{\prime}$ of the original field theory

$$
\mathcal{H}=H_{Q}^{*}\left(\mathcal{H}^{\prime}\right)=\operatorname{ker} Q / \operatorname{im} Q
$$

That is, physical states are annihilated by $Q$ and identified modulo $Q$-exact states. It does not matter which particular operator we choose to represent a class, since a well-known argument shows that spurious states (states of the form $\{Q, \lambda\}$ ) decouple inside physical correlation functions

$$
\left\langle\{Q, \lambda\} \mathcal{O}_{1} \cdots \mathcal{O}_{s}\right\rangle=0, \quad \text { if }\left\{Q, \mathcal{O}_{i}\right\}=0 .
$$

The bracket $\{\cdot, \cdot\}$ represents here either a commutator or an anti-commutator, whichever is appropriate. The existence of the operator $Q$ by itself is of course not enough to warrant 
general covariance. To that end we need another essential ingredient, namely that the energymomentum tensor $T_{\mu \nu}$ is spurious itself

$$
T_{\mu \nu}=\left\{Q, G_{\mu \nu}\right\}
$$

and thus cohomologically trivial. The operator $G_{\mu \nu}$ must be a fermionic rank two tensor. The conserved charges related to $T_{\mu \nu}$ and $G_{\mu \nu}$ are

$$
P_{\mu}=\int T_{\mu 0}, \quad G_{\mu}=\int G_{\mu 0}
$$

where the integrals are taken over a $D-1$ dimensional spacelike surface. These charges form an anti-commuting extension of the translation group

$$
P_{\mu}=\left\{Q, G_{\mu}\right\}, \quad\left\{G_{\mu}, G_{\nu}\right\}=0
$$

that can be viewed as a supersymmetry algebra with charges of spin zero and one, instead of the usual spin one-half.

\subsection{The DESCENT EQUATION}

Regarding the $Q$ symmetry as a spin zero supersymmetry is a very fruitful analogy. In fact, it is convenient to go to a 'superspace' formulation of the theory, since this leads naturally to the non-local observables in the model. In addition to the previous space-time coordinates $x^{\mu}$, we will now have $D$ Grassmannian coordinates $\theta^{\mu}$. Starting from a physical field $\phi(x)$, that we will sometimes denote as $\phi^{(0)}(x)$ to indicate that it is a local operator, the superfield $\Phi(x, \theta)$ is given by

$$
\begin{aligned}
\Phi(x, \theta) & =\exp \theta^{\mu} G_{\mu} \cdot \phi(x) \\
& =\phi^{(0)}(x)+\phi_{\mu}^{(1)}(x) \theta^{\mu}+\ldots+\phi_{\mu_{1} \ldots \mu_{D}}^{(D)}(x) \theta^{\mu_{1}} \ldots \theta^{\mu_{D}} .
\end{aligned}
$$

Here the fields $\phi^{(k)}$ are generated from $\phi^{(0)}$ by repeated application of $G_{\mu}$

$$
\phi_{\mu_{1} \ldots \mu_{k}}^{(k)}(x)=\left\{G_{\mu_{1}},\left\{G_{\mu_{2}}, \ldots,\left\{G_{\mu_{k}}, \phi^{(0)}(x)\right\} \ldots\right\}\right\}
$$

Since $\phi_{\mu_{1} \ldots \mu_{k}}^{(k)}$ is antisymmetric in all its indices, it represents a $k$-form, and we can write (5.44) symbolically as

$$
\left\{G, \phi^{(k)}\right\}=\phi^{(k+1)}
$$


Since by $(5.42)$ we have $\{Q, G\}=d$, these differential forms satisfy the important descent equation

$$
d \phi^{(k)}=\left\{Q, \phi^{(k+1)}\right\}
$$

We can draw two conclusions from this equation. First, it suggests a new class of non-local physical observables. If $C$ is a $k$-dimensional closed submanifold, the descent equation shows that

$$
\phi(C) \equiv \int_{C} \phi^{(k)}(x)
$$

is a physical observable, since

$$
\{Q, \phi(C)\}=\int_{C} d \phi^{(k-1)}=\int_{\partial C} \phi^{(k-1)}=0 .
$$

Secondly, if $C$ and $C^{\prime}$ represent the same class in $H_{k}(M)$, we have $C-C^{\prime}=\partial S$ and

$$
\phi(C)-\phi\left(C^{\prime}\right)=\int_{S} d \phi^{(k)}=\left\{Q, \int_{S} \phi^{(k+1)}\right\}
$$

So the physical observable depends only on the homology class of $C$, as is illustrated in fig. 9. That is, for each class in $H_{k}(M)$ and each element in $\mathcal{H}$ we can construct a non-local operator. In the case of the zero form fields, we have

$$
\phi^{(0)}(x)-\phi^{(0)}(y)=\left\{Q, \int_{x}^{y} d x^{\mu} \phi_{\mu}^{(1)}\right\}
$$

which shows explicitly our claim that the correlators of the local physical operators do not depend on the positions.

\subsection{Perturbations of topological Field theories}

One very important non-local operator, that always exists, is the top-form

$$
\phi(M)=\int_{M} \phi^{(D)}(x)
$$

The importance of thsi particular operators lies in the following fact. Since $\phi^{(D)}$ is a volume form, it can be integrated over the space-time itself. In this way we can make a modification of the action of the topological quantum field theory by adding (5.51) with some coupling coefficient $t$ to the action $S$

$$
S \rightarrow S-t \cdot \int_{M} \phi^{(D)}
$$


while preserving the general covariance. So, the top-dimensional partner of any local observable defines a perturbation of the topological field theory. We can in this way define a multi-parameter family of topological field theories whose partition functions are given by

$$
Z(M, t)=\left\langle\exp \sum_{k} t_{k} \cdot \int_{M} \phi_{k}^{(D)}\right\rangle
$$

The coupling coefficients $t_{k}$ can be seen as a moduli in the space of topological field theories.

If we specialize to the case of two dimensions where $M$ is a surface $\Sigma$, we see that apart from our local observables

$$
\phi(P) \in \mathcal{H}
$$

associated to a point $P \in \Sigma$, we also can consider the operators

$$
\phi\left(S^{1}\right)=\oint \phi^{(1)}, \quad \phi(\Sigma)=\int_{\Sigma} \phi^{(2)}
$$

that correspond to a (non-trivial) cycle and the surface $\Sigma$ itself. Temporarily discarding the one-forms, the most general correlation function in the model is of the form

$$
\left\langle\phi_{i_{1}} \cdots \phi_{i_{s}} \int \phi_{j_{1}}^{(2)} \cdots \int \phi_{j_{r}}^{(2)}\right\rangle_{g}
$$

We have already given away how these correlators can in principle be calculated. One simply considers the more general object

$$
\left\langle\phi_{i_{1}} \cdots \phi_{i_{s}} \exp \sum_{k} t_{k} \int \phi_{k}^{(2)}\right\rangle_{g}
$$

The expansion of this object in the coupling constants $t_{k}$ clearly generates all possible inclusions of two-form operators. However, according to the above reasoning it can alternatively be seen as a correlation function of only the local operators $\phi_{i}$ in a new, perturbed topological field theory, described by the coupling constants $t_{k}$. Since this new theory satisfies all the axioms of $\S 5.3$, all quantities can again be calculated by factorization. That is, all essential information is encoded in the perturbed three-point functions

$$
c_{i j k}(t)=\left\langle\phi_{i} \phi_{j} \phi_{k} \exp \sum_{k} t_{k} \int \phi_{k}^{(2)}\right\rangle_{0}
$$

which define a family of multiplications on the vector space $\mathcal{H}$.

So we finally arrive at the following beautiful picture of (a family of) two-dimensional topological field theories. It is given by a bundle of commutative, associative algebras $\mathcal{H}$ 
fibered over a moduli space $X$. The data $N, \eta$, and $c$ vary continuously and satisfy the relations of $\S 5$.3. Moreover, there is a well-defined map from a state $\phi_{k} \in \mathcal{H}$ to a vector field $\partial / \partial t_{k}$ on $X$. Furthermore, these vector fields commute, and can be integrated to give local coordinates $t_{k}$. In the following section we will give some very explicit examples of all this.

\section{Topological Conformal Field Theory}

We have seen that a general two dimensional topological field theory is solved in terms of the perturbed three-point functions $c_{i j k}(t)$. Our aim will now be to calculate these quantities in some examples. To this end we have to introduce some auxiliary structure: we will impose the condition that the topological field theory be conformally invariant. This might seem a bit superfluous. All physical quantities in a topological field theory are automatically invariant under conformal transformations, since they are independent of the metric. However, we demand that even the non-physical sector - the 'big' Hilbert space $\mathcal{H}^{\prime}$ - be conformally invariant. We will call these models topological conformal field theories (TCFT), see also [17, 30].

\subsection{Twisting $N=2$ Superconformal MODELS}

Conformal invariance requires that the trace of the stress-energy tensor vanishes

$$
T_{\mu}{ }^{\mu}=0
$$

not just within physical correlation functions, but identically as an operator. The stress tensor still has a $Q$ partner $G_{\mu \nu}$

$$
T_{\mu \nu}=\left\{Q, G_{\mu \nu}\right\}
$$

which is also assumed to be traceless

$$
G_{\mu}{ }^{\mu}=0
$$

In complex coordinates $(z, \bar{z})$ the two remaining components $T=T_{z z}$ and $\bar{T}=T_{\bar{z} \bar{z}}$ are respectively holomorphic and anti-holomorphic fields. We will for the moment restrict our attention only to the left-moving fields, i.e., fields holomorphic in $z$. Let us make a list of the different fields that appear in this sector. 
First of all we have the stress tensor $T(z) d z^{2}$ and its $Q$-partner $G(z) d z^{2}$, both quadratic differentials. These have the standard decompositions into modes

$$
T(z)=\sum L_{n} z^{-n-2}, \quad G(z)=\sum G_{n} z^{-n-2}
$$

We will make the further important assumption, that the Hilbert space $\mathcal{H}^{\prime}$ will decompose in highest weight modules of the algebra generated by the $L_{n}$ and $G_{n}$, which is an extension of the $c=0$ Virasoro algebra

$$
\left[L_{m}, L_{n}\right]=(m-n) L_{m+n}
$$

by a vector supersymmetry:

$$
\left[L_{m}, G_{n}\right]=(m-n) G_{m+n}, \quad\left\{G_{m}, G_{n}\right\}=0
$$

The conformal central charge $c$, that in principle could appear in the Virasoro algebra, must necessarily vanish, since a conformal anomaly is not allowed in a general covariant theory, $c f$ critical string theory. We have primary fields $\phi_{i}$ satisfying

$$
L_{n}\left|\phi_{i}\right\rangle=0, \quad G_{n}\left|\phi_{i}\right\rangle=0, \quad(n>0)
$$

Notice the second relation, which is not a consequence of the first. Actually in this respect conformal invariance is somewhat of a misnomer, since we really require invariance under the larger algebra generated by both the stress tensor $T$ and its anti-commuting partner $G$.

There is a second multiplet of fields related to the (anti-commuting) BRST-current $Q(z) d z$, whose conserved charge is the left-moving part of the scalar $Q$-operator

$$
Q=Q_{0}+\bar{Q}_{0}
$$

with

$$
Q_{0}=\oint Q(z) d z, \quad Q(z)=\sum Q_{n} z^{-n-1} .
$$

We will use the notations $Q$ and $Q_{0}$ interchangeably if no confusion arises. Since the BRSTcurrent is a spin one field, it is - by an argument that we will sketch in a moment - necessarily exact:

$$
Q(z)=\{Q, J(z)\}, \quad J(z)=\sum J_{n} z^{-n-1}
$$

where $J(z) d z$ is a bosonic field of spin one, that is, a honest abelian current. So we arrive at the multiplet of holomorphic fields listed in table 1. 


\begin{tabular}{||c||c|c|c|c||}
\hline current & spin $h$ & charge $q$ & $h+\frac{1}{2} q$ & statistics \\
\hline$T(z)$ & 2 & 0 & 2 & boson \\
$G(z)$ & 2 & -1 & $\frac{3}{2}$ & fermion \\
$Q(z)$ & 1 & 1 & $\frac{3}{2}$ & fermion \\
$J(z)$ & 1 & 0 & 1 & boson \\
\hline
\end{tabular}

Table 1: The conserved currents of a topological conformal field theory with their conformal dimensions, charges, and statistics.

All fields $\phi_{i}(z, \bar{z})$ in the theory can be chosen to have some definite conformal dimensions $h_{i}$ and charges $q_{i}$

$$
L_{0}\left|\phi_{i}\right\rangle=h_{i}\left|\phi_{i}\right\rangle, \quad J_{0}\left|\phi_{i}\right\rangle=q_{i}\left|\phi_{i}\right\rangle
$$

Since we have

$$
L_{0}=\left\{Q, G_{0}\right\}, \quad\left[Q, L_{0}\right]=0,
$$

we see immediately that all physical states, satisfying $Q|\phi\rangle=0$, are ground states, with $h=0$. Indeed, suppose $L_{0}|\phi\rangle=h|\phi\rangle$ with $h \neq 0$, then by a familiar argument this state is exact

$$
|\phi\rangle=\frac{1}{h} L_{0}|\phi\rangle=Q|\lambda\rangle, \quad|\lambda\rangle=\frac{1}{h} G_{0}|\phi\rangle .
$$

The multiplet $\{T, G, Q, J\}$ is very reminiscent of the generators of the $N=2$ superconformal algebra, where we would have a stress tensor $T$, two spin $3 / 2$ supercurrents $Q^{+}, Q^{-}$ and a $U(1)$ current $J$. The two sets of currents are related by the 'twist' 60, 61, 68]

$$
T \rightarrow T+\frac{1}{2} \partial J
$$

which gives in particular $L_{0} \rightarrow L_{0}+\frac{1}{2} J_{0}$ and accordingly adds the charge to the conformal dimensions (see table 1)

$$
h \rightarrow h+\frac{1}{2} q .
$$

We can now consider the algebra generated by the modes $L_{n}, G_{n}, Q_{n}, J_{n}$ of the four currents. If they form a closed algebra - which need not be the case, of course - their commutators are completely fixed, due to the uniqueness of the $N=2$ superconformal algebra, that we would obtain after twisting. In this way we arrive at the following algebra 


$$
\begin{array}{cc}
{\left[L_{m}, L_{n}\right]=(m-n) L_{m+n},} & {\left[J_{m}, J_{n}\right]=d \cdot m \delta_{n+m, 0},} \\
{\left[L_{m}, G_{n}\right]=(m-n) G_{m+n},} & {\left[J_{m}, G_{n}\right]=-G_{m+n},} \\
{\left[L_{m}, Q_{n}\right]=-n Q_{m+n},} & {\left[J_{m}, Q_{n}\right]=Q_{m+n},} \\
\left\{G_{m}, Q_{n}\right\}=L_{m+n}+n J_{m+n}+\frac{1}{2} d \cdot m(m+1) \delta_{n+m, 0}, \\
{\left[L_{m}, J_{n}\right]=-n J_{m+n}-\frac{1}{2} d \cdot m(m+1) \delta_{m+n, 0} .}
\end{array}
$$

Most of these relations are self-evident; they simply express the conformal dimensions and charges of the currents. We note the appearance of a central charge $d$ for the $U(1)$ symmetry. In the case of a topological sigma-model $d$ will correspond to the complex dimension of the target space, which explains the choice of symbol. The same constant features as an anomaly in the commutator of the stress tensor with the $U(1)$ current; we will return to this point momentarily. The central charge for the corresponding $N=2$ supersymmetric theory is related to the anomaly in the topological model by $c=3 d$.

The anomaly in the current $J(z)$ leads to selection rules for the correlation functions. $J(z)$ does not transform as a proper current under coordinate transformations, as we read off from the commutation relations of $L_{n}$ with $J_{m}$ in (6.16). If $z \rightarrow w(z)$ denotes a holomorphic coordinate transformation, then

$$
J_{0} \rightarrow J_{0}+d \cdot \oint \partial_{z}\left(\log \partial_{z} w\right)
$$

where the additional term measures the winding number of the map $\partial_{z} w$. So, under the inversion $w=1 / z$ the zero mode $J_{0}$ goes to $J_{0}-d$. This results in a background charge of $-d$ on the sphere. The background charge for arbitrary topology can be found from the covariant expression of the $U(1)$ anomaly

$$
\nabla_{\mu} J^{\mu}=-\frac{d}{8 \pi} \sqrt{h} R
$$

where $h_{\mu \nu}$ is the world-sheet metric and $R$ is the associated scalar curvature. Consequently, since the curvature density integrates to the Euler character $\chi=2-2 g$ on a genus $g$ surface, there is a background charge of $d \cdot(g-1)$ present that has to be compensated. Correlation functions

$$
\left\langle\phi_{i_{1}} \ldots \phi_{i_{s}}\right\rangle_{g}
$$

therefore obey the selection rule

$$
\sum_{i=1}^{s} q_{i}=d(1-g)
$$


In relation to this we note that the two-form operators

$$
\phi_{i}^{(2)}=G_{-1} \bar{G}_{-1} \phi_{i}^{(0)}
$$

have (left, right) charges $\left(q_{i}-1, \bar{q}_{i}-1\right)$.

By the above twisting any $N=2$ superconformal model produces a topological CFT. This not only provides a wealth of examples, but also allows us to use some very interesting results derived in the context of $N=2$ supersymmetry. For instance, the unitary $N=2$ models come with a natural positive inner product, that we can carry over to the topological field theory. Because of the twisting, this inner product is not Lorentz-invariant. In fact, with this inner product we have the hermiticity property $Q^{\dagger}=G_{0}$, which relates a scalar and a vector quantity. By considering the relation

$$
0=\left\langle\phi\left|L_{0}\right| \phi\right\rangle=\| Q|\phi\rangle\left\|^{2}+\right\| G_{0}|\phi\rangle \|^{2}
$$

one shows that in this case the ground states satisfy both

$$
Q|\phi\rangle=0, \quad G_{0}|\phi\rangle=0
$$

The latter condition picks out a unique representative for each $Q$-cohomology class. It should be compared to harmonic forms in ordinary de Rham cohomology theory. In the $N=2$ context these fields are known as chiral primary fields [69.

\subsection{Generalized $S L(2, C)$ invariance and its COnsequences}

We now want to derive an important symmetry property of topological conformal field theories. To this end we recall that the non-local operators can be combined into one superfield 5.43

$$
\Phi(\theta, \bar{\theta})=\phi^{(0)}+\phi^{(1,0)} \theta+\phi^{(0,1)} \bar{\theta}+\phi^{(2)} \theta \bar{\theta} .
$$

Here we suppressed the $(z, \bar{z})$ dependence. Let us now consider a correlation function on the sphere of the form

$$
\left\langle\prod_{i=1}^{s} \int d^{2} z d^{2} \theta \Phi_{i}(z, \bar{z}, \theta, \bar{\theta})\right\rangle_{0} .
$$

This expression is not well-defined, since it is invariant under a fermionic extension of the well-known $S L(2, C)$ symmetry, generated by operators $L_{0}, L_{1}, L_{-1}$ and $G_{0}, G_{1}, G_{-1}$. We 
have to factor out the infinite volume of this group in order to obtain a finite answer. These symmetries correspond to the super-Möbius transformations

$$
z \rightarrow \frac{a z+b}{c z+d}, \quad \theta \rightarrow \frac{\theta+\alpha z^{2}+\beta z+\gamma}{(c z+d)^{2}}
$$

This extended $S L(2, C)$ symmetry can be used to fix three of the $z$-coordinates, say $z_{1}, z_{2}, z_{3}$, at 0,1 , and $\infty$, and put three of the $\theta$-coordinates to zero. We choose these anti-commuting coordinates to be $\theta_{1}, \theta_{2}, \theta_{3}$. If we recall that

$$
\int d^{2} \theta \cdot \Phi=\phi^{(2)},\left.\quad \Phi\right|_{\theta=0}=\phi^{(0)}
$$

then we see that after gauge fixing we are left with a correlation function of the form

$$
\left\langle\phi_{i_{1}}^{(0)} \phi_{i_{2}}^{(0)} \phi_{i_{3}}^{(0)} \int \phi_{i_{4}}^{(2)} \ldots \int \phi_{i_{s}}^{(2)}\right\rangle_{0}
$$

Since we started from an expression that was explicitly symmetric in all indices $i_{1}, \ldots, i_{s}$, this correlator also has this permutational symmetry. That is, it does not matter which three operators we represent as zero-forms - the generalized $S L(2, C)$ invariance tells us that we can interchange a zero and a two-form. An alternative derivation of this result uses the Ward identities associated to this global invariance [17].

In terms of the coefficients $c_{i j k}(t)$ the permutation symmetry of (6.28) gives the important integrability condition

$$
\frac{\partial c_{i j k}}{\partial t_{l}}=\frac{\partial c_{i j l}}{\partial t_{k}}
$$

We stress that this is an additional condition imposed on the family of algebras $c(t)$, and a consequence of the topological invariance. We can integrate this relation three times - at least locally - to find that the three-point functions are actually the third derivatives of a function $F(t)$, the so-called free energy

$$
c_{i j k}(t)=\frac{\partial^{3} F(t)}{\partial t_{i} \partial t_{j} \partial t_{k}} .
$$

Symbolically, $F(t)$ is defined as

$$
F(t)=\left\langle\exp \sum_{i} t_{i} \int \Phi_{i}\right\rangle
$$

that is, $F=\log \tau$, with $\tau(t)$ the string partition function. 
As a corollary to the result (6.29), consider the special identity operator $\phi_{0}=\mathbf{1}$. There is no corresponding two-form, since $G$ commutes with the identity. So the coupling coefficient $t_{0}$ does not exist - there is no modulus associated to $\mathbf{1} \in \mathcal{H}$. This fact, combined with integrability relation (6.29), shows that

$$
0=\frac{\partial c_{i j k}}{\partial t_{0}}=\frac{\partial c_{i j 0}}{\partial t_{k}}=\frac{\partial \eta_{i j}}{\partial t_{k}}
$$

So we have shown that the metric or two-point function $\eta_{i j}$ is independent of the deformation parameters $t_{k}$.

Summarizing, we have derived in this section three additional important results, that followed from the conformal invariance. First, all of the coefficients $c_{i j k}(t)$ are derived from a single function, the free energy $F(t)$. This function is well-defined on local patches of the moduli space of TCFT's. Secondly, the metric $\eta_{i j}(t)$ on the Hilbert space of states is in fact independent of the couplings $t_{k}$. This is equivalent to the statement that $\partial F / \partial t_{0}$ is a quadratic function. Finally, there exists an anomalous $U(1)$ symmetry, which assigns charge $q_{i}$ to the local physical fields $\phi_{i}$, with a background charge $d$, and which corresponds to a scaling relation for the free energy of the form

$$
\sum_{j}\left(q_{j}-1\right) t_{j} \frac{\partial}{\partial t_{j}} F(t)=(d-3) F(t) .
$$

Remarkably enough, these three ingredients will be sufficient to solve the theory in many special cases, in particular for all models with $d<1$ [17].

\subsection{Topological Landau-GinzBurg models}

At this point it might be helpful to consider some examples of TCFT's. The simplest example is a free field theory of $d$ complex bosons and fermions. It can obtained by twisting $d$ copies of the $c=3$ free field realization of the $N=2$ superconformal algebra. The bosons will be written as $x^{i}, x^{\bar{\imath}}$, with $i=1, \ldots, d$. In order to describe the fermions we have to make a choice. This choice is related to the following ambiguity. Up to now we basically only discussed the left-moving sector. Here the topological and the superconformal model were related by the twist

$$
T \rightarrow T \pm \frac{1}{2} \partial J
$$

Here the \pm sign is completely irrelevant, since we have no absolute way to fix the overall sign of the $U(1)$ charge. However, if we combine left and right-movers we can consider either the $(+,+)$ or the $(+,-)$ version. Although the resulting TCFT's are still equivalent, 
since quantum-mechanically we can also not distinguish the signs of the separate left and right-moving currents, the interpretation in terms of the classical geometry of the original superconformal model might be vastly different. In all its generality this phenomenon is known as 'mirror symmetry' [70]. Even in case of our simple free field theory we can consider two different actions and symmetry realizations that lead to equivalent quantum field theories. The problem manifests itself here only in the spin assignments of the fermions. In the $N=2$ model we have four different kinds of fermionic operators that we can denote as

$$
\psi_{L}^{i}(z), \psi_{R}^{i}(\bar{z}), \psi_{L}^{\bar{\imath}}(z), \psi_{R}^{\bar{\imath}}(\bar{z})
$$

These fields, all of spin one-half, obtain the unusual spins of zero and one after twisting. The two possible twists identify as the spin zero fields either the pair $\psi_{L}^{i}, \psi_{R}^{\bar{\imath}}$ or the pair $\psi_{L}^{i}, \psi_{R}^{i}$. We will continue to work with the latter choice, and will combine the two fields into one field

$$
\psi^{i}(z, \bar{z})=\psi_{L}^{i}(z)+\psi_{R}^{i}(\bar{z})
$$

We denote the spin one fields as $\chi_{z}^{\bar{\imath}}, \chi_{\bar{z}}^{\bar{\imath}}$. They are the two components of a one-form $\chi_{\mu}^{\bar{\imath}}$. The action of these fields reads, with a summation over $i=\bar{\imath}$ understood,

$$
S_{0}=\int d^{2} z\left(\partial_{\mu} x^{\bar{\imath}} \partial^{\mu} x^{i}+\chi_{\mu}^{\bar{\tau}} \partial^{\mu} \psi^{i}\right)
$$

The next step is to identify the nilpotent charge $Q$. With $\delta=\{Q, \cdot\}$ the non-vanishing transformations are simply

$$
\delta x^{i}=\psi^{i}, \quad \delta \chi_{\mu}^{\bar{\imath}}=-\partial_{\mu} x^{\bar{\imath}} .
$$

With these field transformations we easily verify that the action is invariant. The stressenergy tensor is indeed $Q$-exact

$$
T(z)=\partial_{z} x^{\bar{\imath}} \partial_{z} x^{i}+\chi_{z}^{\bar{\imath}} \partial_{z} \psi_{L}^{i}=\{Q, G\}, \quad G(z)=-\chi_{z}^{\bar{\imath}} \partial_{z} x^{i}
$$

The set of currents is complemented in this example by

$$
Q(z)=\partial_{z} x^{\bar{\imath}} \psi_{L}^{i}, \quad J(z)=-\chi_{z}^{\bar{\imath}} \psi^{i}
$$

This trivial free field theory can be generalized in two interesting directions. First, we can consider non-linear sigma models, where the flat space-time of one-complex dimension is replaced by an arbitrary $d$-dimensional Ricci-flat Kähler manifold $M$. In superfield notation, with $X^{i}=x^{i}+\theta \psi^{i}+\ldots$, the action reads

$$
S=\int d^{2} z d^{4} \theta \cdot K\left(X^{i}, X^{\bar{\imath}}\right)
$$


where $K$ is the Kähler potential, and $x^{i}, x^{\bar{\imath}}$ are the local coordinates on $M$. These models are well-known to possess $N=2$ superconformal invariance, which translates into topological invariance after twisting. In the above model we simply have chosen the flat target space metric

$$
K\left(X^{i}, X^{\bar{\imath}}\right)=X^{i} X^{\bar{\imath}}
$$

A second possible generalization is the inclusion of a superpotential $W(X)$. In this case we have a so-called topological Landau-Ginzburg model [71], see also [72]. The action reads

$$
S=S_{0}+\int d^{2} z d^{2} \theta \cdot W\left(X^{i}\right)+c . c .
$$

or, after the integration over $(\theta, \bar{\theta})$ and eliminating the auxiliary fields,

$$
\begin{aligned}
S=\int d^{2} z & \left(\partial_{z} x^{\bar{\imath}} \partial_{\bar{z}} x^{i}+\chi_{z}^{\bar{\imath}} \partial_{\bar{z}} \psi_{L}^{i}+\chi_{\bar{z}}^{\bar{\imath}} \partial_{z} \psi_{R}^{i}\right. \\
& \left.+\frac{\partial W}{\partial x^{\bar{\imath}}} \frac{\partial \bar{W}}{\partial x^{i}}+\psi_{L}^{i} \psi_{R}^{j} \frac{\partial^{2} W}{\partial x^{i} \partial x^{j}}+\chi_{z}^{\bar{\imath}} \chi_{\bar{z}}^{\bar{j}} \frac{\partial^{2} \bar{W}}{\partial x^{\bar{\imath}} \partial x^{\bar{\jmath}}}\right) .
\end{aligned}
$$

In this case we have to modify the transformation rules by [71]

$$
\delta \psi_{L}^{i}=\frac{\partial W}{\partial x^{\bar{\imath}}}, \quad \delta \psi_{R}^{i}=-\frac{\partial W}{\partial x^{\bar{i}}}
$$

Landau-Ginzburg models become only conformal invariant at the renormalization group fixed point, if the superpotential is quasi-homogeneous [73, 74]. That is, if there exists a scaling law, with charges $q_{i}$, such that (from now we will use lower index notation $x_{i} \equiv x^{\bar{\imath}}$ to avoid confusion with powers of the coordinates)

$$
W\left(\lambda^{q_{i}} x_{i}\right)=\lambda W\left(x_{i}\right)
$$

Landau-Ginzburg models have the wonderful property that the correlation functions of the chiral primary fields - or, after twisting, the physical fields - are completely determined by the superpotential $W$. In fact, for a quasi-homogeneous potential $W\left(x_{i}\right)$ the local physical fields are simply functions $f\left(x_{i}\right)$ of the invariant field $x_{i} \equiv x^{\bar{\imath}}$. Since we have

$$
\frac{\partial W}{\partial x_{i}}=\left\{Q, \psi_{L}^{i}\right\}
$$

every function of the form $f=g^{i} \partial_{i} W$ is $Q$-exact, and the physical state space is accordingly given by

$$
\mathcal{H}=\frac{\mathbf{C}[x]}{\partial W(x)}
$$


Since there are no singularities in the operator product of two function $f\left(x_{i}\right)$, the operator algebra does not receive corrections, and is also given by (6.48) now considered as a polynomial ring, where $\mathbf{C}[x]$ is the set of polynomials in the $x_{i}$, and we have factored out the ideal generated by

$$
\frac{\partial W}{\partial x_{i}}=0 .
$$

The charge of an element $\phi(x)$ is determined by assigning charge $q_{i}$ to the fundamental field $x_{i}$

In general we can consider deformations of $W$ by an element in $\mathcal{H}$ that preserve the quasihomogeneity, i.e., an operator of charge $q=1$, a modulus or marginal operator. A simple example is the potential

$$
W=x^{3}+y^{3}+z^{3}+a \cdot x y z .
$$

This clearly introduces a modulus - for any value of $a$ the potential is homogeneous, whereas the different rings $\mathcal{H}$ are non-isomorphic. There is a special set of potentials that have no moduli. The corresponding field theories are known as topological minimal modelsthe twisted versions of the minimal $N=2$ superconformal models, and as such were first considered by Eguchi and Yang [68] and, in relation with solvable string theories, by Li [16], In this way the classification of rational singularities enters the subject, since these potentials have an $A D E$ classification, as given in table 2. This is of course the same $A D E$ labeling that features in the classification of modular invariant partition functions in minimal conformal field theories [75].

It is not difficult to give the explicit forms of the rings $\mathcal{H}$. For instance, the $A_{p-1}$ ring with

$$
W(x)=x^{p}
$$

is simply given by

$$
x^{p-1}=0
$$

Thus, a basis is furnished by the elements

$$
\left\{1, x, x^{2}, \ldots, x^{p-2}\right\}
$$

Quite generally the fields $\phi_{i} \in \mathcal{H}$ are in one-to-one correspondence with the exponents of the simply-laced Lie group $G$. The field $\phi_{i}$ has charge $q_{i}=i / p$ with $i+1$ an exponent, and $p=h_{G}$, the dual Coxeter number of $G$. The 'central charge' $d$ of the minimal model is given by

$$
d=\frac{p-2}{p}
$$




\begin{tabular}{|c|c|c|c|}
\hline$G$ & $\Gamma$ & $W$ & $d$ \\
\hline$A_{n}$ & $1 \bullet-\bullet-\cdots-\bullet-\bullet \bullet_{n}$ & $x^{n+1}$ & $\frac{n-1}{n+1}$ \\
\hline$D_{n}$ & i & $x^{n-1}+x y^{2}$ & $\frac{n-2}{n-1}$ \\
\hline$E_{6}$ & $\dot{i}_{--\bullet-\bullet}$ & $x^{3}+y^{4}$ & $\frac{4}{5}$ \\
\hline$E_{7}$ & 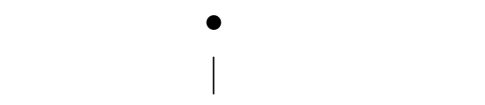 & $x^{3}+x y^{3}$ & $\frac{8}{9}$ \\
\hline$E_{8}$ & $\bullet-\bullet-\bullet-\bullet-\bullet-\bullet-\bullet$ & $x^{3}+y^{5}$ & $\frac{14}{15}$ \\
\hline
\end{tabular}

Table 2: The simply-laced Lie groups $G$, their Dynkin diagrams $\Gamma$, the corresponding superpotentials $W$, and the central charge $d$ of the topological minimal model.

see also table 2. The metric $\eta$ is determined through charge conservation, and is of the skew-diagonal form

$$
\eta_{i j}=\delta_{i+j, p-2}
$$

\subsection{The PERTURBed operator ALGEBra}

It is clear that a choice of superpotential $W(x)$ determines the operator algebra (6.48) and therefore all correlation functions of the local operators $\phi^{(0)}$. As we have explained, by factorization, it suffice to calculate the perturbed three-point function

$$
c_{i j k}(t)=\left\langle\phi_{i} \phi_{j} \phi_{k} \exp \sum_{n} t_{n} \int \phi_{n}^{(2)}\right\rangle_{0}
$$

in order to include also the non-local two form $\phi^{(2)}$. We will be able to calculate this algebra as a result of the existence of a flat metric on the moduli space of TCFT's. Much of the structure we will meet, in particular the existence of 'flat coordinates' associated to a rational singularity, has already appeared in the mathematical literature through the work of K. Saito [76]. Recently, these techniques have been applied in the context of topological field theory [77, 78]. We will sketch here the less sophisticated argument of [17].

For definiteness we will consider only the models in the $A$-series, so that $W(x)=x^{p}$, and 
the basis elements of $\mathcal{H}$ are given by

$$
\phi_{i}=x^{i}, \quad i=0,1, \ldots, p-2
$$

The crucial idea is that the perturbed algebra is still of the form (6.48), but with a more general superpotential

$$
W=x^{p}+\sum_{i=0}^{p-2} g_{i}(t) x^{i} .
$$

Intuitively, a result of this form is to be expected, since we add to the action the operators

$$
\phi_{i}^{(2)}=\int d^{2} \theta \cdot X^{i}
$$

However, the extra terms on the RHS of (6.58) are not of the simple form $t_{i} x^{i}$. This is the case because the operators are only given by $\phi_{i}=x^{i}$ at the conformal $(t=0)$ point, and become more complicated $t$-dependent polynomials after a general perturbation. We therefore should allow for more general functions $g_{i}(t)$ that behave as $g_{i}=t_{i}+\mathcal{O}\left(t^{2}\right)$. For general values of the coupling constants the fields $\phi_{i}$ are defined by

$$
\phi_{i}(x, t)=\frac{\partial W}{\partial t_{i}}=\sum_{j=0}^{p-2} \frac{\partial g_{j}}{\partial t_{i}} x^{j}
$$

We want to calculate the functions $g_{i}(t)$, or equivalently the fields $\phi_{i}(x, t)$.

Of course, one can also consider the $g_{i}$ 's as coordinates on the space of topological field theories, and this is the perfect legitimate point of view taken in [71]. In that case one should worry about contact terms that are quite generally associated to reparametrizations of moduli [80]. The preferred coordinates $t_{k}$, that naturally follow from the conformal invariance underlying the model, are referred to as 'flat coordinates.' The reason for this - not quite appropriate - nomenclature is the following. In $\S 6.2$ we proved that in a general topological conformal field theory the metric

$$
\eta_{i j}=\left\langle\phi_{i} \phi_{j}\right\rangle_{0}
$$

is constant, when consider a function of the moduli $t_{i}$. That is, we always have a flat metric on the space of perturbed TCFT's and we can choose coordinates $t_{i}$ such that this metric is constant. We will use this crucial ingredient to determine the correlation functions. Notice that for a given superpotential $W$ the operator products are completely determined - they are given by the polynomial ring

$$
\phi_{i}(x) \phi_{j}(x)=\sum_{k} c_{i j}{ }^{k} \phi_{k}(x) \quad\left(\bmod W^{\prime}(x)\right) .
$$


The inner product should be compatible with this multiplication, and is thereby uniquely determined to be

$$
\left\langle\phi_{i} \phi_{j}\right\rangle=\oint \frac{d x}{2 \pi i} \frac{\phi_{i}(x) \phi_{j}(x)}{W^{\prime}(x)} .
$$

(Here we used in an essential way the fact that $W$ allows no moduli. Otherwise this metric would only be determined up to a conformal factor. See for instance [79] for an example where this phenomenon occurs.) The above metric clearly respects the operator algebra (6.62), since terms proportional to $W^{\prime}(x)$ do not contribute. It can also be directly derived from the path-integral representation [71]. We know that the fields $\phi_{i}$ should be orthogonal with respect to this inner product. These orthogonal polynomials can be defined in the following elegant way as derivatives of fractional powers of the superpotential

$$
\phi_{i}(x)=\frac{1}{i+1} d W_{+}^{(i+1) / p}
$$

Here $d=\partial / \partial x$, and the + indicates again a truncation to positive powers of $x$, similar as in our discussion of the $\mathrm{KdV}$ hierarchy in $\S 3.1$. One easily verifies, with $z=W^{1 / p}$ that indeed

$$
\begin{aligned}
\left\langle\phi_{i} \phi_{j}\right\rangle & =\frac{1}{2 \pi i} \oint \frac{\left(z^{i} d z\right)_{+}\left(z^{j} d z\right)_{+}}{z^{p-1} d z} \\
& =\frac{1}{2 \pi i} \oint z^{i+j-p+1} d z=\delta_{i+j, p-2}
\end{aligned}
$$

The reader is encouraged to verify that the +'s have legitimately been dropped in this derivation. The solution of the model results if we combine the equations (6.60) and (6.64), and obtain the important result

$$
(i+1) \frac{\partial W}{\partial t_{i}}=\frac{\partial}{\partial x} W_{+}^{(i+1) / p} .
$$

After a relabelling $t_{i} \rightarrow(i+1) t_{i+1}$ this is exactly the equation (4.28) that described the first few 'primary' flows in the $p^{\text {th }} \mathrm{KdV}$ hierarchy. We can identify the fundamental LandauGinzburg field $x$ with the classical momentum $y=D$, and the superpotential $W(x)$ with the classical version of the Lax operator $L(y)$. In this way the operators are related by

$$
\mathcal{O}_{k}=k \cdot \phi_{k-1}=d W_{+}^{k / p}
$$

Some more work is needed to show that also the string partition function and the $\tau$-function coincide. In this way we have recovered a crucial part of the quantum field theory underlying the structures of $\S \S 2-4$. The twisted $N=2$ minimal models where first proposed as the relevant quantum field theories for the $(p, 1)$ string theory by Keke Li [16]. As explained in [18, 19] these minimal models naturally give rise to the top Chern classes that appeared in the intersection numbers defined in $§ 2.4$. 
In case the reader is wondering: equation (6.66) is sufficient to explicitly solve the operators $\phi_{i}(x)$ and the superpotential $W(x)$. The result reads

$$
\phi_{i}(x)=(-1)^{i} \operatorname{det}\left(\begin{array}{ccccc}
-x & 1 & 0 & \cdots & 0 \\
t_{p-2} & -x & 1 & \ddots & \vdots \\
t_{p-3} & t_{p-2} & \ddots & & 0 \\
\vdots & & \ddots & & 1 \\
t_{p-i} & \cdots & t_{p-3} & t_{p-2} & -x
\end{array}\right)
$$

The superpotential is obtained by putting $\partial W / \partial x=\phi_{p-1}$ in the above equation.

\section{Topological String Theory}

In this final section we will start our discussion of topological gravity and topological string theory. Through these vastly more complicated quantum field theories we will be able to make contact with the intersection theory and integrable hierarchies that featured in the first few sections.

There exists a general recipe to associate to a given moduli space a topological quentum field theory whose correlation functions reduce to intersection numbers of cohomology classes of the moduli space 62]. In the case of the moduli space of Riemann surfaces this field theory is known as topological gravity [12]. The fundamental multiplet consists of the spin connection $\omega_{\mu}$, its anti-commuting partner $\psi_{\mu}$, and a bosonic scalar field $\phi$. The BRST transformations read

$$
\delta \omega_{\mu}=\psi_{\mu}, \quad \delta \psi_{\mu}=\partial_{\mu} \phi, \quad \delta \phi=0
$$

The cohomology classes $\sigma_{n}$ correspond to the physical observables

$$
\sigma_{n}=\phi^{n}
$$

The relation with intersection theory is in this formulation conceptually most clear. However, it does not lead easily to explicit calculations. Therefore we choose here a slightly more complicated, but equivalent, representation due to Erik and Herman Verlinde [14, 30]. We will refer to this theory in all its generality as topological string theory.

As in conventional string theory, topological strings will consist of a matter theory coupled to Liouville and ghost fields. The matter sector will be a topological CFT as was studied 
in the previous section. We have seen that the existence of the nilpotent $Q$-charge simplified things enormously. Our aim will be to introduce a dynamical metric, or, after gauge fixing, a Liouville-like field and ghosts, while preserving the $Q$-symmetry at all stages. The fundamental symmetries, generated by the stress-tensor $T_{\mu \nu}$ and its partner $G_{\mu \nu}$, that we encountered in the previous section, will now be lifted to the status of gauge symmetries. In our presentation we will follow very closely [14, 30]. We will explain the construction starting with our most simple example.

\subsection{ACTION AND SYMMETRIES}

Let us reconsider the $d=1$ topological sigma model of $\S 6.3$ whose action we now write for a general world-sheet metric $g_{\mu \nu}$ as

$$
S=\int d^{2} z \sqrt{g} g^{\mu \nu}\left(\partial_{\mu} x \partial_{\nu} \bar{x}+\chi_{\mu} \partial_{\nu} \psi\right)
$$

We want to couple this model to gravity, so that the metric $g_{\mu \nu}$ becomes dynamical, without ruining the topological invariance guaranteed by the $Q$-symmetry. Therefore, each field must have a fermionic partner. So, we introduce the metric's fermionic partner $\psi_{\mu \nu}$ together with the transformation rules

$$
\delta g_{\mu \nu}=\psi_{\mu \nu}, \quad \delta \psi_{\mu \nu}=0 .
$$

Just as variations of the action with respect to the metric give the stress-energy tensor, variations with respect to $\psi_{\mu \nu}$ give its partner $G_{\mu \nu}$

$$
T^{\mu \nu}=\frac{1}{\sqrt{g}} \frac{\delta S}{\delta g_{\mu \nu}}, \quad G^{\mu \nu}=\frac{1}{\sqrt{g}} \frac{\delta S}{\delta \psi_{\mu \nu}} .
$$

We already found that $G_{\mu \nu}$ was given by $\chi_{\mu} \partial_{\nu} \psi$. These functional derivatives can be integrated to get the full action describing the coupling of topological matter to topological gravity

$$
S_{m}=\int d^{2} z \sqrt{g} g^{\mu \nu}\left(\partial_{\mu} x \partial_{\nu} \bar{x}+\chi_{\mu} \partial_{\nu} \psi+\psi_{\mu \rho} \chi^{\rho} \partial_{\nu} x\right) .
$$

We will work in the conformal gauge

$$
g_{\mu \nu}=e^{\phi} \delta_{\mu \nu}, \quad \psi_{\mu \nu}=\rho e^{\phi} \delta_{\mu \nu}
$$

which requires us to introduce the bosonic (commuting) ghosts $\beta$ and $\gamma$ in addition to the fermionic (anticommuting) ghosts $b$ and $c$. The ghosts $b$ and $\beta$ have spin two, whereas $c$ and $\gamma$ have spin -1 . Of course, the central charge of this ghost multiplet is again $c=0$, by our 
familiar theme of 'bosons cancel fermions.' It is an easy exercise to write down the generators $T, G, Q$, and $J$ for the ghost system, and verify that they obey the relations we have come to expect.

In terms of the conformal factors $\phi$ and $\rho$ the $Q$-transformation of the metric reads

$$
\delta \phi=\rho, \quad \delta \rho=0
$$

Since the total central charge vanishes, we can fix the Weyl transformations that shift these modes by the conditions $\partial \bar{\partial} \phi=\widehat{R}$ and $\partial \bar{\partial} \rho=0$, where $\widehat{R}$ is the world-sheet curvature of a fiducial background metric $\hat{g}_{\mu \nu}$. These constraints will be implemented with the aid of Lagrange multipliers $\pi$ and $\lambda$. Taking all together, the total action for the theory is

$$
\begin{gathered}
S=S_{m}+S_{g h}+S_{L}, \\
S_{g h}=\int d^{2} z(b \bar{\partial} c+\beta \bar{\partial} \gamma+\text { c.c. }) \\
S_{L}=\int d^{2} z(\pi(\partial \bar{\partial} \phi-\widehat{R})-\lambda \partial \bar{\partial} \rho) .
\end{gathered}
$$

In order to find the physical spectrum we have to know the correct BRST charge. In fact, here we have two candidates. First, we have a topological field theory that comes naturally with a nilpotent $Q$ charge - we have been very careful to preserve this feature. Secondly, we are dealing with a gauge theory complete with ghosts and its own BRST charge, since we gauged the symmetries generated by the stress tensor $T$ and its fermionic partner $G$. This BRST charge is of the form

$$
Q_{B R S T}=\oint \frac{d z}{2 \pi i}: c\left(T_{m}+T_{L}+\frac{1}{2} T_{g h}\right)+\gamma\left(G_{m}+G_{L}+\frac{1}{2} G_{g h}\right):
$$

and is a simple generalization of the well-known $Q_{B R S T}$ of bosonic string theory. A precise analysis shows that in this case the correct BRST charge, whose cohomology determines the spectrum of the theory, is the sum of these two charges

$$
Q_{\text {total }}=Q+Q_{B R S T}
$$

An analysis, that we will not repeat here, shows that the physical operators with respect to $Q_{\text {total }}$ are of the form

$$
\mathcal{O}=\phi_{i} \cdot \tilde{\sigma}_{n}
$$

where $\phi_{i}$ is a matter field with charge $q_{i}$ and $\tilde{\sigma}_{n}$ is a combination of ghost and Liouville fields with charge $n$, a non-negative integer. These gravitational fields $\tilde{\sigma}_{n}$ are a new feature 
of topological string theory - they will of course correspond to the Mumford-Morita-Miller classes of $\S 2$.

We now must prescribe how to calculate correlation functions of these operators. As in critical string theory, we define the higher genus correlation functions so that they become volume forms on the moduli space of Riemann surfaces. These forms are then integrated to get the final correlation functions, using the Beltrami differentials $\mu_{1}, \ldots, \mu_{3 g-3}$. The precise definition is

$$
\left\langle\mathcal{O}_{1} \ldots \mathcal{O}_{n}\right\rangle_{g} \equiv \int_{\mathcal{M}_{g}}\left\langle\int \mathcal{O}_{1}^{(2)} \ldots \int \mathcal{O}_{n}^{(2)} \prod_{a=1}^{3 g-3} \int \mu_{a} G \int \bar{\mu}_{a} \bar{G}\right\rangle_{g} .
$$

The operators are of the form $\mathcal{O}_{k}=\phi_{i_{k}} \cdot \tilde{\sigma}_{n_{k}}$, and satisfy the selection rule

$$
\sum_{k}\left(n_{k}+q_{i_{k}}-1\right)=(d-3)(1-g)
$$

The total background charge on the RHS is composed of the matter contribution $d(1-g)$ and a term needed to cancel the charge of the $3 g-3 G$-insertions. The correlation function (7.13) is a generalization of the one for critical string theory, with the anti-ghosts $b(z)$ are replaced by the supercurrents $G(z)$, see [30] for further comments on this analogy.

\subsection{Pure Topological Gravity}

We will proceed to solve topological string theory in the case of pure topological gravity. That is, the case that no matter sector is present $(d=0)$. Since we work with a cohomological theory, there are many possible representations of the operators. We will follow here [14] and choose a representation in which the correlation functions receive no contribution from the interior of moduli space.

Let us start with the definition of the operators $\tilde{\sigma}_{n}$

$$
\tilde{\sigma}_{n}=3^{n} e^{\frac{2}{3}(n-1) \pi}(\tilde{\gamma})^{n}, \quad \text { with } \quad \tilde{\gamma}=\bar{\partial}\left(\frac{1}{2} \partial c+c \partial \phi-\text { c.c. }\right)
$$

In order to understand this definition a bit more intuitively, introduce $Q=Q_{0}+\bar{Q}_{0}$ and $Q_{-}=Q_{0}-\bar{Q}_{0}$. Since these operators have an interpretation as Dolbeault operators $\partial, \bar{\partial}$ on the world-sheet, one can verify that

$$
\tilde{\gamma}=\left\{Q,\left\{Q_{-}, \phi\right\}\right\} \sim \partial \bar{\partial} \phi=\widehat{R}
$$

So $\tilde{\gamma}$ is roughly the curvature, and $\tilde{\sigma}_{n}$ corresponds to the $n^{\text {th }}$ power of $\widehat{R}$. A much precise analysis [14, 30] shows that, in the notation of $\S 2$, the operator $\tilde{\sigma}_{n}$ is related to the cohomology 
class $\sigma_{n} \in H^{*}\left(\mathcal{M}_{g, s}\right)$ by

$$
\tilde{\sigma}_{n}=(2 n+1) ! ! \cdot \sigma_{n}=\mathcal{O}_{2 n+1} .
$$

We note that $\tilde{\gamma}$ naively appears to decouple because it is BRST exact. However, the model is not well-defined as it stands. To define the correlation functions unambiguously, the fields must be restricted to a subspace $\mathcal{H}_{0}$ of the 'big' Hilbert space $\mathcal{H}^{\prime}$

$$
\mathcal{H}_{0} \equiv\left\{|\phi\rangle \in \mathcal{H}^{\prime} ;\left(b_{0}-\bar{b}_{0}\right)|\phi\rangle=0\right\}
$$

otherwise, there is an obstruction to globally define correlation function over $\mathcal{M}_{g, s} 81$. So the physical Hilbert space $\mathcal{H}=H_{Q}^{*}\left(\mathcal{H}_{0}\right)$ is composed of $Q$ cohomology classes equivariant with respect to $b_{0}-\bar{b}_{0}$. This insures that local coordinates can be consistently defined at the punctures. It can easily be seen that, even though $\tilde{\gamma}$ is BRST exact in $\mathcal{H}^{\prime}$, it is not in $\mathcal{H}_{0}$ and therefore does not decouple.

\subsection{CONTACT AND FACtORIZATion terms}

As was shown in [14] the correlation functions for pure topological gravity are completely determined from the boundary of moduli space. In fact, contact terms, which occur whenever two operators collide and are quite generally of the form

$$
\mathcal{O}_{i}(z) \cdot \mathcal{O}_{j}(0) \sim \mathcal{O}_{k}(0) \delta^{2}(z)
$$

suffice to solve the model. To see this we must work out the contact term algebra explicitly. We will sketch the argument of [14].

Define the states $\left|\tilde{\sigma}_{m}\right\rangle=\tilde{\sigma}_{m}(0)|0\rangle$, and consider the contribution of the operator $\tilde{\sigma}_{n}$ when it is close to the operator $\tilde{\sigma}_{m}$, located at $z=0$ in some coordinates

$$
\int d^{2} z \cdot \tilde{\sigma}_{n}(z, \bar{z}) \cdot \tilde{\sigma}_{m}(0)|0\rangle=\int_{|q|<\epsilon} \frac{d^{2} q}{|q|^{2}} G_{0} \bar{G}_{0} q^{L_{0}} \bar{q}^{L_{0}} \tilde{\sigma}_{n}(1)\left|\tilde{\sigma}_{m}\right\rangle .
$$

Now recall that $\tilde{\sigma}_{n} \sim \tilde{\gamma}^{n}$ with $\tilde{\gamma}=\left\{Q,\left\{Q_{-}, \phi\right\}\right\}$. Since $L_{0}$ is $Q$ exact, we find a contribution due to

$$
\int d^{2} q \partial_{q} \partial_{\bar{q}} q^{L_{0}} \bar{q}^{\bar{L}_{0}} \phi(0)\left|\tilde{\sigma}_{n+m-1}\right\rangle
$$

where $q^{L_{0}} \bar{q}^{\bar{L}_{0}} \phi(0) \sim \log |q|^{2}+$ regular, because of the anomalous covariance of the field $\phi$. This clearly gives a $\delta$-function contribution, and with the inclusion of all factors one finds in the end

$$
\int d^{2} z \tilde{\sigma}_{n}(z, \bar{z}) \tilde{\sigma}_{m}(0)=(2 m+1) \tilde{\sigma}_{n+m-1}(0)
$$


Note that this expression is not symmetric in $n$ and $m$,

$$
\int\left[\tilde{\sigma}_{n}, \tilde{\sigma}_{m}\right]=2(m-n) \tilde{\sigma}_{m+n-1}
$$

where we see the appearance of a Virasoro algebra.

Let us now consider a general correlator of the form

$$
\left\langle\tilde{\sigma}_{n} \tilde{\sigma}_{k_{1}} \ldots \tilde{\sigma}_{k_{s}}\right\rangle_{g}
$$

We want to study the contributions to this amplitude that we obtain if we integrate the two-form $\tilde{\sigma}_{n}$ over the Riemann surface. In general there are three different contributions: the contact terms that we examined above, factorization terms, and, finally, bulk terms. The factorization terms arise when the surface itself degenerates - the cases (ii) and (iii) of fig. 2. They may be expressed as sums over factorized correlation functions with $\tilde{\sigma}_{i}$ insertions, and are, by charge conservation, of the general form

$$
\sum_{i+j=n-2} A_{i j}^{(n)}\left\langle\tilde{\sigma}_{i} \tilde{\sigma}_{k_{1}} \ldots \tilde{\sigma}_{k_{r}}\right\rangle_{g^{\prime}}\left\langle\tilde{\sigma}_{j} \tilde{\sigma}_{k_{r+1}} \ldots \tilde{\sigma}_{k_{s}}\right\rangle_{g-g^{\prime}}
$$

or

$$
\sum_{i+j=n-2} B_{i j}^{(n)}\left\langle\tilde{\sigma}_{i} \tilde{\sigma}_{j} \tilde{\sigma}_{k_{1}} \ldots \tilde{\sigma}_{k_{s}}\right\rangle_{g-1}
$$

with some coefficients $A_{i j}^{(n)}, B_{i j}^{(n)}$. The bulk terms describe the contributions from the interior of moduli space. Quite remarkably, one can show that these contributions are absent due to the asymmetry in the contact terms. Also the factorization terms are completely determined by consistency: $A_{i j}^{(n)}=B_{i j}^{(n)}=\frac{1}{2}$. So, in the end we are left with the following recursion relations for the correlation functions of topological gravity.

$$
\begin{gathered}
\left\langle\tilde{\sigma}_{n} \prod_{k \in S} \tilde{\sigma}_{k}\right\rangle_{g}=\sum_{k \in S}(2 k+1)\left\langle\tilde{\sigma}_{n+k-1} \prod_{k^{\prime} \neq k} \tilde{\sigma}_{k^{\prime}}\right\rangle_{g}+\sum_{i+j=n-2} \frac{1}{2}\left\langle\tilde{\sigma}_{i} \tilde{\sigma}_{j} \prod \tilde{\sigma}_{k}\right\rangle_{g-1} \\
+\sum_{\substack{g^{\prime} ; S=X \cup Y \\
i+j=n-2}} \frac{1}{2}\left\langle\tilde{\sigma}_{i} \prod_{k \in X} \tilde{\sigma}_{k}\right\rangle_{g^{\prime}}\left\langle\tilde{\sigma}_{j} \prod_{k \in Y} \tilde{\sigma}_{k}\right\rangle_{g-g^{\prime}} .
\end{gathered}
$$

Here $S=\left\{k_{1}, \ldots, k_{s}\right\}$, and the last summation is over all partitions of $S$ into two subsets $X, Y$. The three terms on the RHS are associated to the three inequivalent degenerations of fig. 2: contact terms and factorization terms of a homology cycle or dividing cycle. The contributions always come from the boundary of moduli space, so the recursion relation lowers the genus $g$ and/or the number of operators $s$, and ultimately gives factors of the three-point function on the sphere,

$$
\left\langle\tilde{\sigma}_{0}^{3}\right\rangle_{0}=\left\langle\sigma_{0}^{3}\right\rangle_{0}=1,
$$


or the one-point function on the torus

$$
\left\langle\tilde{\sigma}_{1}\right\rangle_{1}=3\left\langle\sigma_{1}\right\rangle_{1}=\frac{1}{8}
$$

Therefore the recursion relations suffice to calculate any intersection number. In the case $n=0$ the relation (7.27) is nothing but the puncture equation (2.13); the case $n=1$ is a linear combination of the dilaton equation (2.15) and the ghost charge conservation (2.8). Note that in these two cases the factorization terms are absent.

Although these relations are manifestly non-linear in terms of the free energy $F$, they turn out to be linear when written in terms of the partition function $\tau=e^{F}$, due to the wonderful relation

$$
\tau^{-1} \partial^{2} \tau=\partial^{2} F+(\partial F)^{2}
$$

Indeed, consider the partition function

$$
\tau(\tilde{t})=\exp \sum_{g=0}^{\infty}\left\langle\exp \sum_{n} \tilde{t}_{n} \tilde{\sigma}_{n}\right\rangle_{g}
$$

where the coupling coefficients $\tilde{t}_{n}$ are related to the $\mathrm{KdV}$ times by $\tilde{t}_{n}=t_{2 n+1}$. Now, if we recall that a derivative $\partial \tau / \partial \tilde{t}_{n}$ corresponds to an insertion of $\tilde{\sigma}_{n}$, whereas a multiplication $\tilde{t}_{n} \cdot \tau$ removes the same operator from the generating function, the recursion relations (7.27) can be expressed as linear, homogeneous differential equations for the $\tau$-function

$$
L_{n} \cdot \tau=0, \quad(n \geq-1)
$$

where $L_{n}$ denote the differential operators

$$
\begin{aligned}
L_{-1} & =-\frac{1}{2} \frac{\partial}{\partial \tilde{t}_{0}}+\sum_{k=1}^{\infty}\left(k+\frac{1}{2}\right) \tilde{t}_{k} \frac{\partial}{\partial \tilde{t}_{k-1}}+\frac{1}{4} \tilde{t}_{0}^{2} \\
L_{0} & =-\frac{1}{2} \frac{\partial}{\partial \tilde{t}_{1}}+\sum_{k=0}^{\infty}\left(k+\frac{1}{2}\right) \tilde{t}_{k} \frac{\partial}{\partial \tilde{t}_{k}}+\frac{1}{16}, \\
L_{n} & =-\frac{1}{2} \frac{\partial}{\partial \tilde{t}_{n-1}}+\sum_{k=0}^{\infty}\left(k+\frac{1}{2}\right) \tilde{t}_{k} \frac{\partial}{\partial \tilde{t}_{k+n}}+\frac{1}{4} \sum_{i=1}^{n} \frac{\partial^{2}}{\partial \tilde{t}_{i-1} \partial \tilde{t}_{n-i}} .
\end{aligned}
$$

These are exactly the equations that according to $\S 4.4$ characterize the $\tau$-function of the $\mathrm{KdV}$ hierarchy that we studied in such great detail before. So, the above manipulations amount to a sketch of a quantum field theory proof of Witten's original conjecture.

In the more general case of a topological minimal model coupled to gravity, these Virasoro constraints are replaced by the $W$-constraints

$$
W_{n}^{(s)} \cdot \tau=0, \quad s=2, \ldots, p, n \geq 1-s,
$$


The differential operators $W_{n}^{(s)}$ form a $W$-algebra associated to the Lie group $G$ that labeled the minimal model. They are spin $s$ currents that generalize the Virasoro algebra. We have one $W$-generator for each Casimir in the group $G$. The constraints now obtain an interpretation as multiple contact and factorization terms [24, 16]. A full discussion of these relations is however completely outside the scope of these lectures.

\section{Acknowledgements}

I wish to thank the organizers of this wonderful school for inviting me to present these lectures and for creating such a stimulating and enjoyable atmosphere. Needless to say I benefitted greatly from countless insightful discussion with my collaborators Erik Verlinde, Herman Verlinde, and Edward Witten. I further thank Rob Rudd for his assistance in preparing a preliminary version of these notes.

\section{References}

[1] V. Kazakov, Phys. Lett. 159B (1985) 303; F. David, Nucl. Phys. B257 (1985) 45; V. Kazakov, I. Kostov, and A. Migdal, Phys. Lett. 157B (1985) 295; J. Fröhlich, The statistical mechanics of surfaces, in Applications of Field Theory to Statistical Mechanics, L. Garrido ed. (Springer, 1985).

[2] F. David, Phys. Lett. 159B (1985) 303; D. Boulatov, V. Kazakov, and A. Migdal, Nucl. Phys. B275 [FS117] (1986) 543; A. Billoire and F. David, Phys. Lett. 186B (1986) 279; J. Jurkievic, A. Krzywicki, and B. Peterson, Phys. Lett. 186B (1986) 273; J. Ambjorn, B. Durhuus, J. Fröhlich, and P. Orland, Nucl. Phys. B270 [FS16] (1986) 457.

[3] V. Kazakov, Mod. Phys. Lett. A4 (1989) 2125.

[4] E. Brézin and V. Kazakov, Exactly solvable field theories of closed strings, Phys. Lett. B236 (1990) 144 .

M. Douglas and S. Shenker, Strings in less than one dimension, Nucl. Phys. B335 (1990) 635. D.J. Gross and A. Migdal, Nonperturbative two dimensional quantum gravity, Phys. Rev. Lett. 64 (1990) 127.

[5] D.J. Gross and A. Migdal, A nonperturbative treatment of two dimensional quantum gravity, Nucl. Phys. B340 (1990) 333.

T. Banks, M. Douglas, N. Seiberg, and S. Shenker, Microscopic and macroscopic loops in nonperturbative two dimensional gravity, Phys. Lett. 238B (1990) 279.

[6] E. Brézin, M. Douglas, V. Kazakov, and S. Shenker, The Ising model coupled to 2d gravity: a nonperturbative analysis, Phys. Lett. 237B (1990) 43

D.J. Gross and A. Migdal, Nonperturbative solution of the Ising model on a random surface, Phys. Rev. Lett. 64 (1990) 717.

C. Crnković, P. Ginsparg, and G. Moore, The Ising model, the Yang-Lee edge singularity, and 2d quantum gravity, Phys. Lett. 237B (1990) 196.

[7] M. Douglas, Strings in less than one dimension and the generalized KdV hierarchies, Phys. Lett. 238B (1990) 176. 
[8] E. Brézin, V. Kazakov, and Al.B. Zamolodchikov, Scaling violation in a field theory of closed strings in one physical dimension, Nucl. Phys. B338 (1990).

D.J. Gross and N. Miljković, A nonperturbative solution of $D=1$ string theory, Phys. Lett. 238B (1990) 217.

P. Ginsparg and J. Zinn-Justin, 2-d Gravity and 1-d matter, Phys. Lett. 240B (1990) 333.

D.J. Gross and I.R. Klebanov, One-dimensional string theory on a circle, Nucl. Phys. B344 (1990) 475.

[9] Random Surfaces, Quantum Gravity and Strings, proceedings of the 1990 Cargèse workshop, O. Alvarez, E. Marinari, and P. Windey eds. (plenum Press, 1991).

D.J. Gross, Nonperturbative string theory, in the Proceedings of the International Colloquium on Modern Quantum Field Theory, Bombay, 1990.

I.R. Klebanov, String theory in two dimensions, lectures at 1991 Trieste Spring School on String Theory and Quantum Gravity, Princeton preprint PUPT-1271 (July, 1991).

P. Ginsparg, Matrix models of 2d gravity, lectures at 1991 Trieste Summer School, Los Alamos preprint (December, 1991).

[10] E. Witten, On the topological phase of two dimensional gravity, Nucl. Phys. B340 (1990) 281.

[11] E. Witten, Two dimensional gravity and intersection theory on moduli space, Surveys In Diff. Geom. 1 (1991) 243.

[12] J. Labastida, M. Pernici, and E. Witten, Topological gravity in two dimensions, Nucl. Phys. B310 (1988) 611.

D. Montano and J. Sonnenschein, The topology of moduli space and quantum field theory, Nucl. Phys. B313 (1989) 258; Nucl. Phys. 324 (1990) 348.

R. Myers and V. Periwal, Topological gravity and moduli space, Nucl. Phys. 333 (1990) 536.

[13] J. Distler, 2d quantum gravity, topological field theory and the multicritical matrix models, Nucl. Phys. B342 (1990) 523.

[14] E. Verlinde and H. Verlinde, A solution of two-dimensional topological quantum gravity, Nucl. Phys. B348 (1991) 457.

[15] R. Dijkgraaf and E. Witten, Mean field theory, topological field theory, and multi-matrix models, Nucl. Phys. B342 (1990) 486.

[16] K. Li, Topological gravity with minimal matter; Recursion relations in topological gravity with minimal matter, Nucl. Phys. 354 (1991) 711, 725.

[17] R. Dijkgraaf, E. Verlinde, and H. Verlinde, Topological strings in $d<1$, Nucl. Phys. B352 (1991) 59.

[18] E. Witten, The $N$ matrix model and gauged WZW models, IAS preprint IASSNS-HEP-91/26 (June, 1991).

[19] E. Witten, Algebraic geometry associated with matrix models of two dimensional gravity, IAS preprint IASSNS-HEP-91/74 (October, 1991).

[20] M. Kontsevich, Intersection theory on the moduli space of curves, Funk. Anal. i Pril. 25 (1991) 50 (in Russian).

[21] M. Kontsevich, Intersection theory on the moduli space of curves and the matrix Airy function, 30 Arbeitstagung Bonn, Max-Planck-Institut preprint MPI/91-47.

[22] M. Kontsevich, Intersection theory on the moduli space of curves and the matrix Airy function, Max-Planck-Institut preprint MPI/91-77. 
[23] E. Witten, Lectures at IAS, Fall 1991.

[24] R. Dijkgraaf, E. Verlinde, and H. Verlinde, Loop equations and Virasoro constraints in nonperturbative 2d quantum gravity, Nucl. Phys. B348 (1991) 435.

[25] M. Fukuma, H. Kawai, and R. Nakayama, Continuum Schwinger-Dyson equations and universal structures in two-dimensional quantum gravity, Int. J. Mod. Phys. A6 (1991) 1385.

[26] B.H. Lian and G.J. Zuckerman, New selection rules and physical states in 2d gravity: conformal gauge, Phys. Lett. 254 (1991) 417.

[27] S. Kharchev, A. Marschakov, A. Mironov, A. Morozov, A. Zabrodin, Unification of all string models with $c<1$, Lebedev Institute preprint FIAN/TD-9/91.

[28] C. Itzykson and J.-B. Zuber, Combinatorics of the Modular Gro up II: The Kontsevich Integrals, Saclay preprint SPhT/92-001, submitted to Int. J. Mod. Phys.

[29] G. Segal and G. Wilson, Loop groups and equations of KdV type, Publ. Math. I.H.E.S. 61 (1985) 1.

[30] R. Dijkgraaf, E. Verlinde, and H. Verlinde, Notes on topological string theory and 2d quantum gravity, in String Theory and Quantum Gravity, Proceedings of the Trieste Spring School 1990, M. Green et al. eds. (World-Scientific, 1991).

[31] P. Deligne and M. Mumford, The irreducibility of the space of curves of given genus, Publ. I.H.E.S. 45 (1969) 75.

[32] D. Mumford, Towards an enumerative geometry of the moduli space of curves, in Arithmetic and Geometry, M. Artin and J. Tate eds. (Birkhäuser, Basel, 1983).

[33] S. Morita, Characteristic classes of surface bundles, Invent. Math. 90 (1987) 551.

[34] E. Miller, The homology of the mapping class group, J. Diff. Geom. 24 (1986) 1.

[35] P. Deligne, Letter to E. Witten (October, 1989).

[36] J. Horne, Intersection theory and two dimensional gravity at genus 3 and 4, Mod. Phys. Lett. A5 (1990) 2127.

[37] C. Faber, Chow rings of moduli spaces of curves: I and II, Ann. Math. 132 (1990) 331; 421.

[38] K. Strebel, Quadratic Differentials (Springer-Verlag, 1984).

[39] R.C. Penner, The decorated Teichmüller space of punctured surfaces, Commun. Math. Phys. 113 (1987) 299; Perturbative series and the moduli space of Riemann surfaces, J. Diff. Geom. 27 (1988) 35.

[40] G. 't Hooft, Nucl. Phys. B72 (1974) 461.

[41] J. Harer and D. Zagier, The Euler characteristic of the moduli space of curves, Invent. Math. 185 (1986) 457.

[42] I.M. Gelfand and L.A. Dikii, The resolvent and Hamiltonian systems, Funct. Anal. Appl. 11:2 (1977) 93.

[43] V.E. Zakharov, Funk. Anal. i Pril. 14 (1980) 89.

[44] I. Krichever, Topological minimal models and dispersionless Lax equation, Turin preprint, Commun. Math. Phys. to appear (1991); Topological minimal models and soliton equations, Landau Institute preprint (1991). 
[45] M. Sato, Soliton equations as dynamical systems on infinite dimensional Grassmann manifolds, RIMS Kokyuroku 439 (1981) 30.

M. Sato and Y. Sato, Soliton equations as dynamical systems in an infinite dimensional Grassmannian, in Nonlinear Partial Differential Equations in Applied Sciences, P.Lax, H. Fujita, and G. Strang eds. (North-Holland, Amsterdam, 1982).

[46] E. Date, M. Jimbo, M. Kashiwara, T. Miwa, Transformation groups for soliton equations, RIMS Symp. Nonlinear Integrable Systems - Classical Theory and Quantum Theory (World Scientific, Singapore, 1983).

[47] T. Miwa, Proc. Jap. Acd. 58A (1982) 9.

[48] G. Moore, Geometry of the string equations, Commun. Math. Phys. 133 (1990) 261; Matrix models of $2 d$ gravity and isomonodromic deformation, in Random Surfaces, Quantum Gravity and Strings, Proceedings of the 1990 Cargèse workshop, O. Alvarez, E. Marinari, and P. Windey eds. (Plenum Press, 1991).

[49] M. Adler and P. van Moerbeke, The $W_{p}$-gravity version of the Witten-Kontsevich model, Brandeis preprint (September, 1991).

[50] Harish-Chandra, Differential operators on a semisimple Lie algebra, Am. J. Math. 79 (1987) 87.

[51] M.L. Mehta, A method of integration over matrix variables, Commun. Math. Phys. 79 (1981) 327.

[52] C. Itzykson and J.-B. Zuber, The planar approximation II, J. Math. Phys. 21 (1980) 411.

[53] R.F. Picken, The Duistermaat-Heckman integration formula on flag manifolds, J. Math. Phys. B31 (1990) 616.

[54] A. Miranov, A. Morozov, Phys. Lett. B252 (1990) 47. Yu. Makeenko, A. Marshakov, A. Mironov, A. Morozov, Nucl. Phys. 356 (1991) 574. H.S. La, Commun. Math. Phys. 140 (1991) 569. A.M. Semikhatov, Lebedev Institute preprints.

[55] J. Goeree, W constraints in 2-d quantum gravity, Nucl. Phys. B358 (1991) 73

[56] M. Fukuma, H. Kawai, and R. Nakayama, Infinite dimensional Grassmannian structure of two-dimensional quantum gravity, University of Tokyo preprint UT-572 (November, 1990).

[57] E. Witten, On the Kontsevich model and other models of two dimensional gravity, IAS preprint IASSNS-HEP-91/24 (June, 1991).

[58] D.J. Gross and M. Newman, Unitary and Hermitian matrices in an external field II: the Kontsevich model and continuum Virasoro constraints, Princeton preprint (December, 1991).

[59] A. Marschakov, A. Mironov, and A. Morozov, On equivalence of topological and quantum 2d gravity, Lebedev Institute preprint FIAN/TD/04-91.

[60] E. Witten, Topological quantum field theory, Commun. Math. Phys. 117 (1988) 353.

[61] E. Witten, Topological sigma models, Commun. Math. Phys. 118 (1988) 411.

[62] E. Witten, Introduction to cohomological field theories, Int. J. Mod. Phys. A6 (1991) 2775.

[63] P. van Baal, An introduction to topological Yang-Mills theory, Acta Phys. Polon. B21 (1990) 73.

[64] D. Birmingham, M. Blau, M. Rakowski, and G. Thompson, Topological field theory, preprint CERN-TH 6045/91, to be published in Physics Reports.

[65] M.F. Atiyah, Topological quantum field theories, Publ. Math. I.H.E.S. 68 (1988) 175. 
[66] R. Dijkgraaf, A geometrical approach to two-dimensional conformal field theory, Ph.D. Thesis (Utrecht, 1989).

[67] S. Shenker, The strength of nonperturbative effects in string theory, in Random Surfaces, Quantum Gravity and Strings, Proceedings of the 1990 Cargèse workshop, O. Alvarez, E. Marinari, and P. Windey eds. (Plenum Press, 1991).

[68] T. Eguchi and S.-K. Yang, $N=2$ superconformal models as topological field theories, Mod. Phys. Lett A5 (1990) 1693.

[69] W. Lerche, C. Vafa, and N.P. Warner, Chiral rings in $N=2$ superconformal theories, Nucl. Phys B324 (1989) 427.

[70] B. Greene and R. Plesser, Duality in Calabi-Yau moduli space, Nucl. Phys. B338 (1990) 15.

[71] C. Vafa, Topological Landau-Ginzburg models, Mod. Phys. Lett. A6 (1990) 337.

[72] K. Ito, Topological phase of $N=2$ superconformal field theory and topological Landau-Ginzburg field theories, Phys. Lett. 250 (1990) 91.

[73] E. Martinec, Algebraic geometry and effective lagrangians, Phys. Lett. 217B (1989) 431; Criticality, catastrophe and compactifications, in V.G. Knizhnik memorial volume, 1989.

[74] C. Vafa and N. Warner, Catastrophes and the classification of conformal field theories, Phys. Lett. 218B (1989) 51.

[75] A. Cappelli, C. Itzykson, and J.-B. Zuber, The ADE classification of minimal and $A_{1}^{(1)}$ conformal theories, Commun. Math. Phys. 113 (1987) 1.

[76] K. Saito, Publ. RIMS 19 (1983) 1231.

M. Saito, Ann. Inst. Fourier 39:1 (1989) 27.

[77] B. Blok and A. Varchenko, Topological conformal field theories and the flat coordinates, IAS preprint IASSNS-HEP-91/5 (January, 1991).

[78] W. Lerche, D.J. Smit, and N.P. Warner, Differential equations for periods and flat coordinates in two-dimensional matter theories, Preprint UCB-PTH-91-39 (July, 1991).

[79] E. Verlinde and N.P. Warner, Topological Landau-Ginzburg matter at $c=3$, IAS preprint IASSNS-HEP-91-16 (March, 1991).

[80] D. Kutasov, Geometry on the space of conformal field theories and contact terms, Phys. Lett B220 (1989) 153.

[81] J. Distler and P. Nelson, Topological couplings and contact terms in 2d field theory, Commun. Math. Phys. 138 (1991) 273. 